\title{
Visualization of Cranial Motor Neurons in Live Transgenic Zebrafish Expressing Green Fluorescent Protein Under the Control of the Islet-1 Promoter/Enhancer
}

\author{
Shin-ichi Higashijima, ${ }^{1,2}$ Yoshiki Hotta, ${ }^{3}$ and Hitoshi Okamoto ${ }^{4}$ \\ 1/nheritance and Variation Group, Precursory Research for Embryonic Science and Technology, Japan Science and \\ Technology Corporation, Honmachi, Kawaguchi, Saitama 332-0012, Japan, 2Division of Morphogenesis, National \\ Institute for Basic Biology, Myodaijicho, Okazaki, Aichi 444-8585, Japan, 3National Institute of Genetics, Mishima, \\ Shizuoka 411-8540, Japan, and 4Laboratory for Developmental Gene Regulation, Brain Science Institute, RIKEN \\ (The Institute of Physical and Chemical Research), Hirosawa, Wako, Saitama 351-0198, Japan
}

\begin{abstract}
We generated germ line-transmitting transgenic zebrafish that express green fluorescent protein (GFP) in the cranial motor neurons. This was accomplished by fusing GFP sequences to Islet-1 promoter/enhancer sequences that were sufficient for neural-specific expression. The expression of GFP by the motor neurons in the transgenic fish enabled visualization of the cell bodies, main axons, and the peripheral branches within the muscles. GFP-labeled motor neurons could be followed at high resolution for at least up to day four, when most larval neural circuits become functional, and larvae begin to swim and capture prey. Using this line, we analyzed axonal outgrowth by the
\end{abstract}

Developing motor neurons in the vertebrate hindbrain are an attractive system in which to study mechanisms of segmentation and the processes regulating specific axonal pathfinding (Guthrie, 1996; Lumsden and Krumlauf, 1996; Pfaff and Kintner, 1998). Chick and mouse are the most frequently used experimental model organisms to study motor neuron development in vertebrates. Recently, the zebrafish has emerged as another useful model system. Several features of zebrafish make them especially amenable for developmental studies, including development of the hindbrain. The embryos are transparent, making it possible to readily visualize internal structures and cells in living zebrafish. The embryonic hindbrain is relatively simple and manipulable

Received July 14, 1999; revised Oct. 6, 1999; accepted Oct. 14, 1999.

This work was supported by grants from the Japan Science and Technology Corporation, the Ministry of Education, Science, and Culture of Japan, and Special Coordination Fund from Science and Technology Agency of Japan. We especially thank N. Ueno, in whose laboratory the majority of this study was performed. We thank G. Eguchi and G. Mandel for generous support. We thank Y. Ishikawa, K. Kawamura, and S. Kuratani for their suggestions on the anatomy of cranial motor and sensory neurons. We thank G. Mandel, J. Fetcho, K. Cho, A. Thomson, Y. Ishikawa, and J. Y. Kuwada for comments on this manuscript. We thank M. Sugiura for assistance with the experiments; K. Takamatsu and Y. Katoh for maintaining our fish colony; M. Nikaido for instruction in the technique of mosaic analysis; K. Umesono for the donation of a modified GFP; M. Petkovich, A. Picker, and $\mathrm{H}$. Takeda for the donation of zebrafish genomic libraries; and members of the Ueno laboratory for encouragement and discussion. The zn8 (= zn5) antibody was obtained from the Developmental Studies Hybridoma Bank maintained by the Department of Biology at the University of Iowa.

Correspondence should be addressed to Hitoshi Okamoto, Laboratory for Developmental Gene Regulation, Brain Science Institute, RIKEN (The Institute of Physical and Chemical Research), 2-1 Hirosawa, Wako, Saitama 351-0198, Japan. E-mail: hitoshi@brain.riken.go.jp.

Dr. Higashijima's present address: Department of Neurobiology and Behavior, State University of New York at Stony Brook, Stony Brook, NY 11794. E-mail: shigashijima@notes.cc.sunysb.edu.

Copyright (C) 1999 Society for Neuroscience $0270-6474 / 00 / 200206-13 \$ 15.00 / 0$ cranial motor neurons. Furthermore, by selective application of Dil to specific GFP-positive nerve branches, we showed that the two clusters of trigeminal motor neurons in rhombomeres 2 and 3 innervate different peripheral targets. This finding suggests that the trigeminal motor neurons in the two clusters adopt distinct fates. In future experiments, this transgenic line of zebrafish will allow for a genetic analysis of cranial motor neuron development.

Key words: zebrafish; neuron-specific promoter; transgenic; Islet-1; motor neuron; GFP; live visualization
(Chandrasekhar et al., 1997, 1998), and the zebrafish is amenable to large-scale genetic studies (Driever et al., 1996; Haffter et al., 1996) that have generated interesting mutations in hindbrain motor neurons (Chandrasekhar et al., 1999).

Despite these potential advantages of zebrafish for the study of motor neuron differentiation in the hindbrain, this system has not yet been fully exploited. No one has ever successfully visualized motor neurons and their axons in live embryos, which would allow a dynamic analysis of migration by differentiating motor neurons and pathfinding by their extending growth cones. The ability to observe the dynamics of neuronal differentiation may facilitate the detection of the effects of genetic mutation or other molecular and cellular manipulations that might otherwise be missed. In Drosophila melanogaster and Caenorhabditis elegans, reproducible labeling of specific types of neurons in vivo by expression of GFP under the control of a cell-type-specific promoter has been used as a powerful tool to study development of those neurons (Murray et al., 1998; Zallen et al., 1998). In vertebrates, however, this approach has only been successful under limited conditions (Dynes and Ngai, 1998; Okada et al., 1999), partly because of a lack of appropriate promoters for cell-type-specific expression of GFP or because of difficulty in observation caused by opacity and complexity of the brain.

Recently, two groups have shown that transgenic zebrafish expressing GFP in specific tissues can be reliably generated (Higashijima et al., 1997b; Long et al., 1997). In the present study, we applied this approach to generate zebrafish in which cranial motor neurons could be selectively visualized to provide a new tool for studying motor neuron development. Our strategy was to use the Islet-1 (Isl1) gene to drive GFP expression in cranial motor neurons. Isl1 is a member of the LIM/homeobox gene 


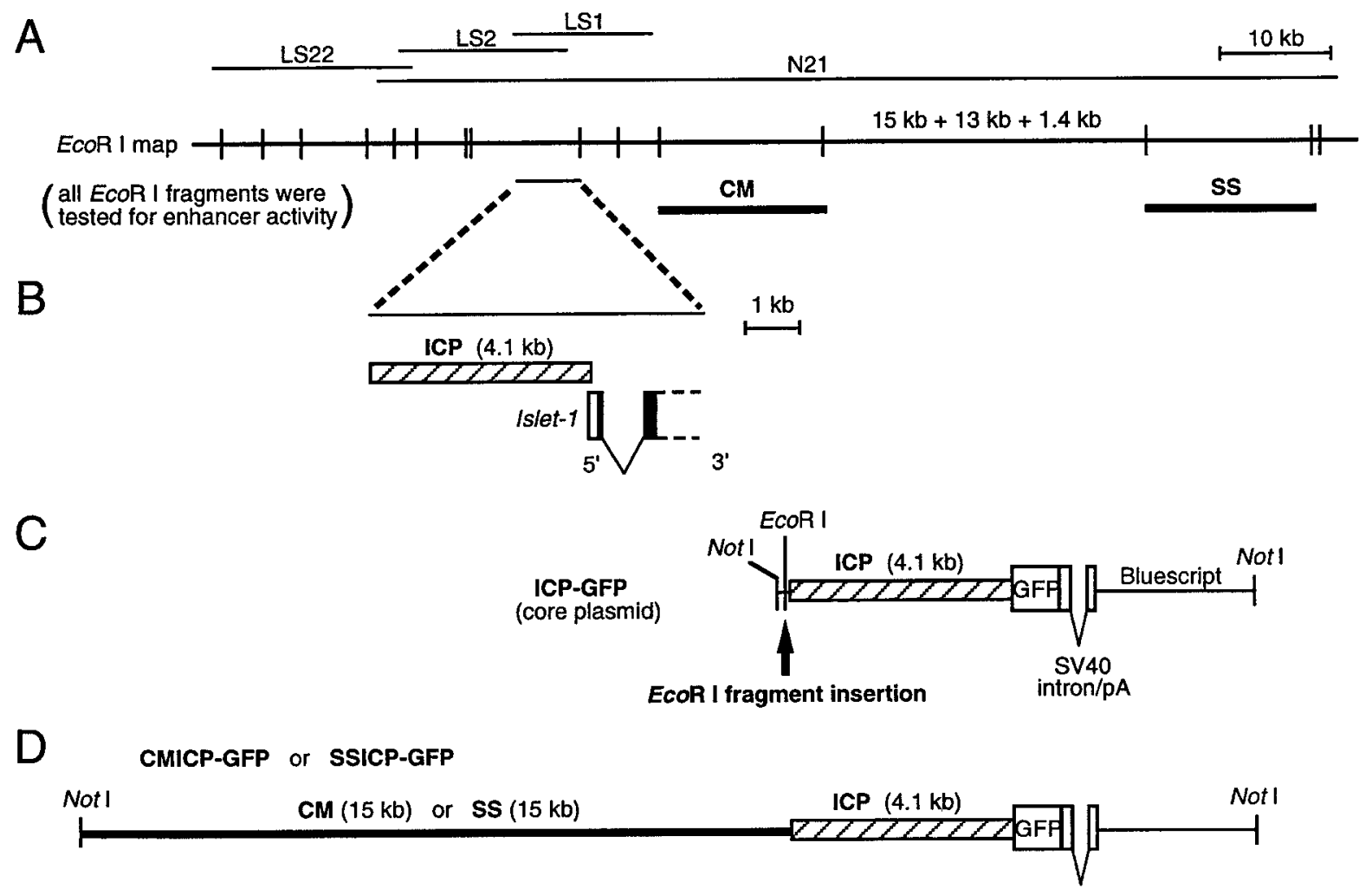

Figure 1. Genomic structure of the Islet-1 (Isl1) gene and the plasmid constructs. A, EcoRI restriction map of the genomic DNA flanking the Isl1 gene. Lines over the map represent phage clones ( $L S 1, L S 2$, and $L S 22)$ and a BAC clone (N21). The thick line, CM, includes the enhancer elements for driving GFP expression in cranial motor neurons, whereas $S S$ includes the enhancer elements for driving GFP expression in the trigeminal ganglion cells and Rohon-Beard cells. Other EcoRI fragments were negative for enhancer activity by our transient expression assay. $B$, Close-up view of the genomic region around the Isl1 promoter. Filled boxes are protein coding regions. The hatched box represents the putative Isl1 promoter $(I C P)$, which contains $\sim 4.1 \mathrm{~kb}$ of the $5^{\prime}$ upstream region and an $\sim 30$ bp of the $5^{\prime}$ untranslated region of the Isl 1 gene. It was used in all the constructs as a core promoter. $C$, A map of the ICP-GFP, the core plasmid. Every genomic EcoRI fragment was inserted into the EcoRI site located in the immediate upstream of ICP. NotI was used to linearize the plasmid DNA for microinjection. $D$, A map of the CMICP-GFP or SSICP-GFP (containing the SS-fragment) plasmid. The CMICP-GFP plasmid drove expression of GFP in the cranial motor neurons, and it was used for generating the Isl1-GFP line. The SSICP-GFP plasmid drove expression of GFP both in Rohon-Beard neurons and the trigeminal sensory neurons.

family and is expressed in all postmitotic motor neurons early in their development (Ericson et al., 1992; Korzh et al., 1993; Inoue et al., 1994; Tsuchida et al., 1994; Appel et al., 1995; Tokumoto et al., 1995; Varela-Echavarría et al., 1996; Osumi et al., 1997). Thus, we expected that the promoter/enhancer of the Isl1 gene would be able to drive GFP expression in cranial motor neurons. We cloned genomic fragments of the zebrafish Isl 1 gene and identified DNA sequences that could direct expression of GFP in the cranial motor neurons of DNA-injected embryos. We then established a stable transgenic line (Isl1-GFP) of zebrafish that expressed GFP in the cranial motor neurons.

The intensity of GFP fluorescence by the cranial motor neurons in the Isl1-GFP line was sufficiently high to enable clear visualization of the cell bodies, main axons, and the peripheral branches in the target muscles. In addition to cranial motor neurons, GFP was expressed in the cranial sensory neurons, except for those in the trigeminal ganglion. The generation of this transgenic line and the characterization of the GFP-expressing neurons set the stage for a genetic dissection of the development of these neurons in future experiments.

As an initial illustration of the usefulness of the Isl1-GFP line, we characterized in detail the development of the trigeminal motor neurons. We asked whether the rhombomeric location of the trigeminal motor neurons correlated with the targets inner- vated by their axons. This was analyzed by backlabeling trigeminal motor neurons with selective DiI application to specific trigeminal nerve branches that could be seen because of their GFP fluorescence. We found that trigeminal neurons from rhombomeres 2 and 3 innervate different target muscles. This result, together with our developmental analysis of the trigeminal motor neurons, suggests that trigeminal neurons generated in $\mathrm{r} 2$ and $\mathrm{r} 3$ adopt distinct fates, supporting the idea that segmental origin dictates the fate of the two populations of trigeminal motor neurons.

\section{MATERIALS AND METHODS}

Cloning of the Isl1 genomic DNA, construction of plasmids, and microinjection of DNA into zebrafish embryos. The Isl1 cDNA was used to screen a series of zebrafish genomic phage libraries. An initial screening and two rounds of genome walking identified a genomic region of $\sim 40 \mathrm{~kb}$ in total (Fig. 1A, LS1, LS2, LS22). Screening of a zebrafish BAC library was performed by Genome Systems, and one BAC clone (Fig. 1A, N21), 90 $\mathrm{kb}$ in length, was isolated. To examine the putative transcriptional start site of the Isl1 gene, RACE (rapid amplification of cDNA ends)-PCR was performed as described previously (Higashijima et al., 1997a) using two Isl1-specific primers. The results suggested that the Isll cDNA (Inoue et al., 1994) was almost full-length. Sequence analysis of the corresponding genomic DNA suggested that there are no further introns other than the one shown in Figure $1 B$ in the $5^{\prime}$ region of the $I s l 1$ gene. A fragment of DNA corresponding to the 5' half of LS1 was PCR- 
amplified. This fragment, termed the putative Isl 1 core promoter (ICP), contained $4.1 \mathrm{~kb}$ of $5^{\prime}$ upstream region and $\sim 30 \mathrm{bp}$ of $5^{\prime}$ untranslated region of the Isl 1 gene. The fragment had, at the $5^{\prime}$ end, an EcoRI site derived from the $\lambda$ DASHII (Stratagene, La Jolla, CA) phage vector sequence, and a KpnI site at the $3^{\prime}$ end, which was introduced with the PCR primer. The ICP-GFP plasmid was generated in the following manner. The backbone plasmid was pBluescript II SK (Stratagene). The NotI site was converted to an NcoI site, and then, the KpnI site was converted to an NotI site. Synthetic oligonucleotides were used in these experiments. A fragment containing an SV40 poly(A) signal with an intron was extracted from the pcDNA1 plasmid (Invitrogen, San Diego, CA) and introduced between the XbaI and NcoI sites of the modified pBluescript II SK. The resultant plasmid was digested with SpeI and $X b a \mathrm{I}$ and self-ligated to disrupt these sites. Then, the NcoI site at the $3^{\prime}$ end of the insert was converted to an $X b a \mathrm{I}$ site using a synthetic oligonucleotide. The HindIII-BglII fragment encoding the modified GFP (codon-humanized, S65A, Y145F; kindly provided by K. Umesono, Kyoto University) was PCR-amplified, and introduced between the HindIII site and BamHI site of the resultant plasmid. This GFP-encoding fragment carried a $K p n I$ site upstream of the initiation codon. Then, the ClaI site within the remaining multiple cloning site of this plasmid was converted to an Eco RI site using a synthetic oligonucleotide. Finally, the ICP-GFP plasmid was created by inserting the ICP fragment between the EcoRI and $K p n I$ sites immediately upstream of the GFP-encoding region. The ICP-GFP plasmid therefore had unique NotI and EcoRI sites just $5^{\prime}$ to the ICP (Fig. $1 C$ ). All the recognizable EcoRI fragments derived from the cloned region of the Isl1 genomic DNA were individually subcloned into the EcoRI site of the ICP-GFP plasmid. Each plasmid DNA was linearized by NotI, which is located upstream of the EcoRI cloning site and used for microinjection. Preparation of DNA and microinjection was performed essentially as described previously (Higashijima et al., 1997b). Linearized plasmid DNA was extracted using phenol-chloroform and then chloroform, precipitated by ethanol, and dissolved in distilled water. For injection of DNA solution, a holder supporting a glass micropipette with a beveled opening was connected to a $10 \mathrm{ml}$ disposable plastic syringe through a plastic tube. A DNA solution of $\sim 25 \mathrm{ng} / \mathrm{ml}$ was injected into the cytoplasm of a one-cell-stage zebrafish embryo with its chorion intact under dissecting microscope by manually adding air pressure to the syringe. We adjusted the injection volume so that the injected solution spread in a sphere with its diameter ranging from one quarter to one half that of the cytoplasm. For the transient expression assay, the injection volume was empirically adjusted such that, on average, approximately one-fourth of the injected embryos died or became malformed by the next day. When the injected embryos were raised to adulthood to generate germ line-transmitting fish, the injection volume was increased such that one-third to one-half of the injected embryos died or were malformed by the next day. Expression of GFP was examined on the first and second days of development. Three of 113 injected fish turned to be the founders for the transgenic progeny. The frequency of transgenic zebrafish successfully generated using the CMICP-GFP construct was significantly lower than in a previous study when we used constructs that drive GFP expression under the control of the $\alpha$-actin promoter (20\%; Higashijima et al., 1997b). Although the exact reason for this remains unknown, we may have missed transgenic lines with low-level GFP expression.

Embryos and staging. Zebrafish were maintained as described in Westerfield (1995). Most analyses were performed using homozygous transgenic embryos from the Isl1-GFP line. The embryos were collected after crossing homozygous parents. In the early phase of the experiments, homozygous embryos were obtained by crossing heterozygous parents. Homozygous fish exhibited no abnormality or retardation in the development of the nervous system. Embryos were collected within 30 min after fertilization and were allowed to develop at $28.5^{\circ} \mathrm{C}$ to the required age, in uncrowded conditions. The developmental age of the embryos corresponds to the number of hours elapsed since fertilization. Morphological features of the embryos in our experiments at a given time stage were, in general, consistent with the zebrafish standard staging table (Kimmel et al., 1995). Because repeated anesthetizations are suggested to retard development (Kimmel et al., 1995), each embryo was examined only once for most of the experiments. To reduce pigmentation, embryos were transferred to fish water containing $0.002 \%$ of phenylthiourea (PTU; Nakarai) between 12 and $22 \mathrm{hr}$ (Burrill and Easter, 1994). The concentration of PTU was reduced to $0.001 \%$ at $48 \mathrm{hr}$, to $0.0003 \%$ at $72 \mathrm{hr}$, and to $0.0001 \%$ at $96 \mathrm{hr}$. At this concentration of PTU, no abnormality in development was observed.
Fluorescent microscopic observations. Initial examination was performed using an epifluorescence dissecting microscope with a GFP plus filter (Leica, Nussloch, Germany). A PlanApo 1.6× objective lens was used. For detailed examination, fluorescent embryos were anesthetized using $0.01 \%$ ethyl- $m$-aminobenzoate methanesulphonate (Sigma, St. Louis, MO) and mounted in 1.2\% agar (Difco, Detroit, MI) on a depression slide glass, as described in Westerfield (1995). Embryos were either examined under a Zeiss (Oberkochen, Germany) upright epifluorescence microscope with an FITC or a GFP (Chroma 41015) filter cassette or under a Zeiss 510 confocal microscope (510-CLSM, upright) with $488 \mathrm{~nm}$ excitation and a $505-550 \mathrm{~nm}$ bandpass filter. Most observations, except for those of rhodamine-phalloidin or antibody-labeled samples, were performed using live material because visibility was best in living fish. A Plan-Neofluar $20 \times$ [numerical aperture (NA) 0.5] objective lens was used for most observations. For higher magnifications, waterimmersion lenses, Achroplan $63 \times($ NA 0.9) and $100 \times($ NA 1.0), were used. For three-dimensional (3-D) reconstruction of the images, serial optical sections at $\sim 4 \mu \mathrm{m}$ intervals $(20 \times$ objective) or $0.5-1.5-\mu \mathrm{m}$ intervals (63 and $100 \times$ objective) were taken, and $3-\mathrm{D}$ images were reconstructed from the stacked confocal images by the software supplied with the 510-CLSM.

For DiI-, Cy3- and rhodamine-labeled samples, observations were performed using the 510-CLSM with $568 \mathrm{~nm}$ excitation and a 585 longpass filter. For 2-p-dimethylaminostyrylpyridylethyl iodide (DASPEI)-labeled samples, $488 \mathrm{~nm}$ excitation and a 585 longpass filter were used. A DASPEI signal was also apparent during the collection of images for GFP (505-550 nm bandpass). For 3-D reconstruction of images for signals from GFP and other dyes, serial optical sections were obtained either simultaneously or sequentially. Each image was pseudocolored (GFP, green; others, red) and compiled.

Mosaic analysis. Cells derived from the homozygous Isl1-GFP embryos were transplanted into wild-type hosts at the sphere stage as in Westerfield (1995). Donor cells were transplanted near the animal pole of host embryos to increase the chance that progeny of donor cells would be distributed in the cranial region of the host embryo. Mosaic embryos and larvae were examined between 48 and $90 \mathrm{hr}$.

Retrograde labeling of the trigeminal motor neurons. Larvae were anesthetized at $\sim 72-90 \mathrm{hr}$ and embedded in $1.2 \%$ agar on a glass slide in an orientation that allowed access to the nerve to be labeled. The agar overlying the injection site was removed. The fluorescent lipophilic dye DiI (Molecular Probes, Eugene, OR), at $2 \mathrm{mg} / \mathrm{ml}$ in ethanol was pressure-injected (Eppendorf Transjector 5246) into a region around a labeled nerve under an epifluorescent dissection microscope with the longpass GFP filter (see above). Fluorescence signals derived from both GFP (green) and DiI (orange) were simultaneously observed under this condition. The injected larvae were allowed to develop at room temperature for $4 \mathrm{hr}$ so that the DiI would retrogradely label the hindbrain neurons. DiI injection was also performed in fixed embryos. In this case, the injected embryos were incubated in $4 \%$ paraformaldehyde in PBS at room temperature for $12 \mathrm{hr}$.

In situ hybridization, antibody staining, rhodamine-phalloidin staining, and DASPEI live staining. In situ hybridization and antibody staining were performed according to standard methods (Westerfield, 1995). Isl1 cDNA (Inoue et al., 1994), zn5 antibody (Trevarrow et al., 1990), and Cy3-conjugated anti-mouse antibody (Jackson ImmunoResearch, West Grove, PA) were used. For rhodamine-phalloidin staining, larvae were fixed and treated in acetone as described previously (Westerfield, 1995). The larvae were washed in PBS containing 1\% dimethylsulfoxide and $0.2 \%$ Triton-X 100 , and incubated in $0.0002 \mathrm{U} / \mathrm{ml}$ rhodamine-phalloidin (Molecular Probes) in the same solution for $1 \mathrm{hr}$ at room temperature with shaking. They were observed after washing. For DASPEI staining, larvae were immersed in $0.1 \mathrm{~mm}$ DASPEI (Molecular Probes) in E3 (in mM: $5 \mathrm{NaCl}, 0.17 \mathrm{KCl}, 0.33 \mathrm{CaCl}_{2}$, and $0.33 \mathrm{MgSO}_{4}$ ) for $20 \mathrm{~min}$, as in Balak et al. (1990) and Whitfield et al. (1996), and observed after rinsing.

\section{RESULTS}

\section{Identification of the Is/1 promoter/enhancer region and generation of transgenic zebrafish}

The Isll gene and its protein product have been used as early markers for motor neurons in vertebrates. However, the promoter/enhancer region that directs gene expression in motor neurons has not yet been identified in any vertebrate species. To isolate the promoter/enhancer of the Isl1 gene that is sufficient for 

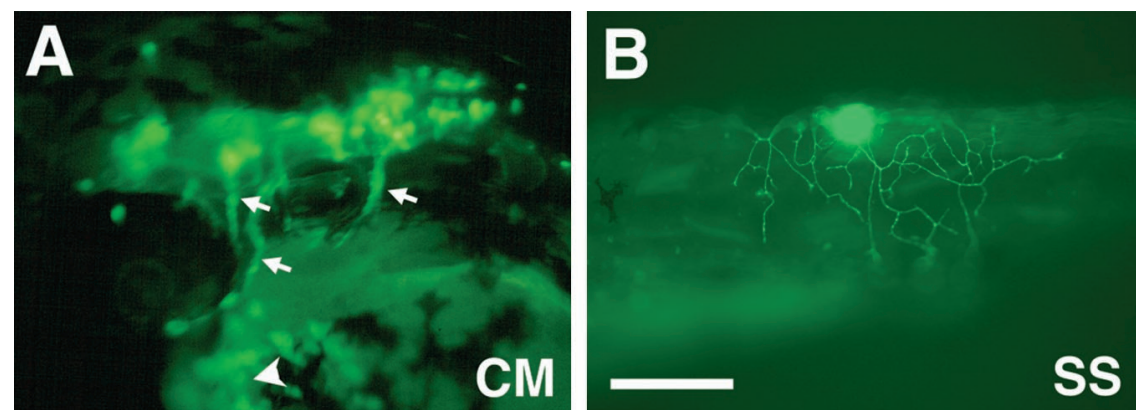

Figure 2. Zebrafish Isl1 promoter/enhancer activity in transient expression assays. $A$, GFP expression in the head of a $40 \mathrm{hr}$ embryo injected with the CMICPGFP plasmid. The arrows indicate GFP expression in branchial motor nerves. The arrowhead indicates GFP expression in the hatching gland cells. Anterior is to the left, dorsal is up. B, A lateral view of GFP expression in a Rohon-Beard cell in the spinal cord of a 36 hr embryo injected with the SSICP-GFP plasmid. Scale bar: $A, 200 \mu \mathrm{m} ; B, 230 \mu \mathrm{m}$. expression in zebrafish motor neurons, $\sim 100 \mathrm{~kb}$ genomic sequences flanking the $I s l 1$ gene were isolated by screening both $\lambda$ phage and BAC zebrafish genomic libraries (Fig. 1A). The structure of the $5^{\prime}$ region of the $I s l 1$ gene is shown in Figure $1 B$. The core plasmid, ICP-GFP, was generated by fusing $4.1 \mathrm{~kb}$ of the Isl1 promoter, ICP, to the gene encoding a modified GFP (Fig. $1 C$ ). When the ICP-GFP DNA was injected into embryos, GFP was expressed by hatching gland cells (data not shown) whose precursors normally expresses the Isll gene (Inoue et al., 1994), but not by motor neurons. This suggests that ICP includes en- hancer elements that regulate hatching gland expression of the Isl1 gene.

To identify enhancer elements that control expression in motor neurons, a series of constructs were generated by introducing genomic EcoRI fragments into the ICP-GFP plasmid (Fig. $1 C$ ). All recognizable EcoRI fragments in the cloned region (Fig. 1A) were tested for their enhancer activity by injecting each construct into embryos. In the injected embryos, GFP expression was frequently observed in the hatching gland cells presumably because of the core promoter (ICP) activity that was common to all
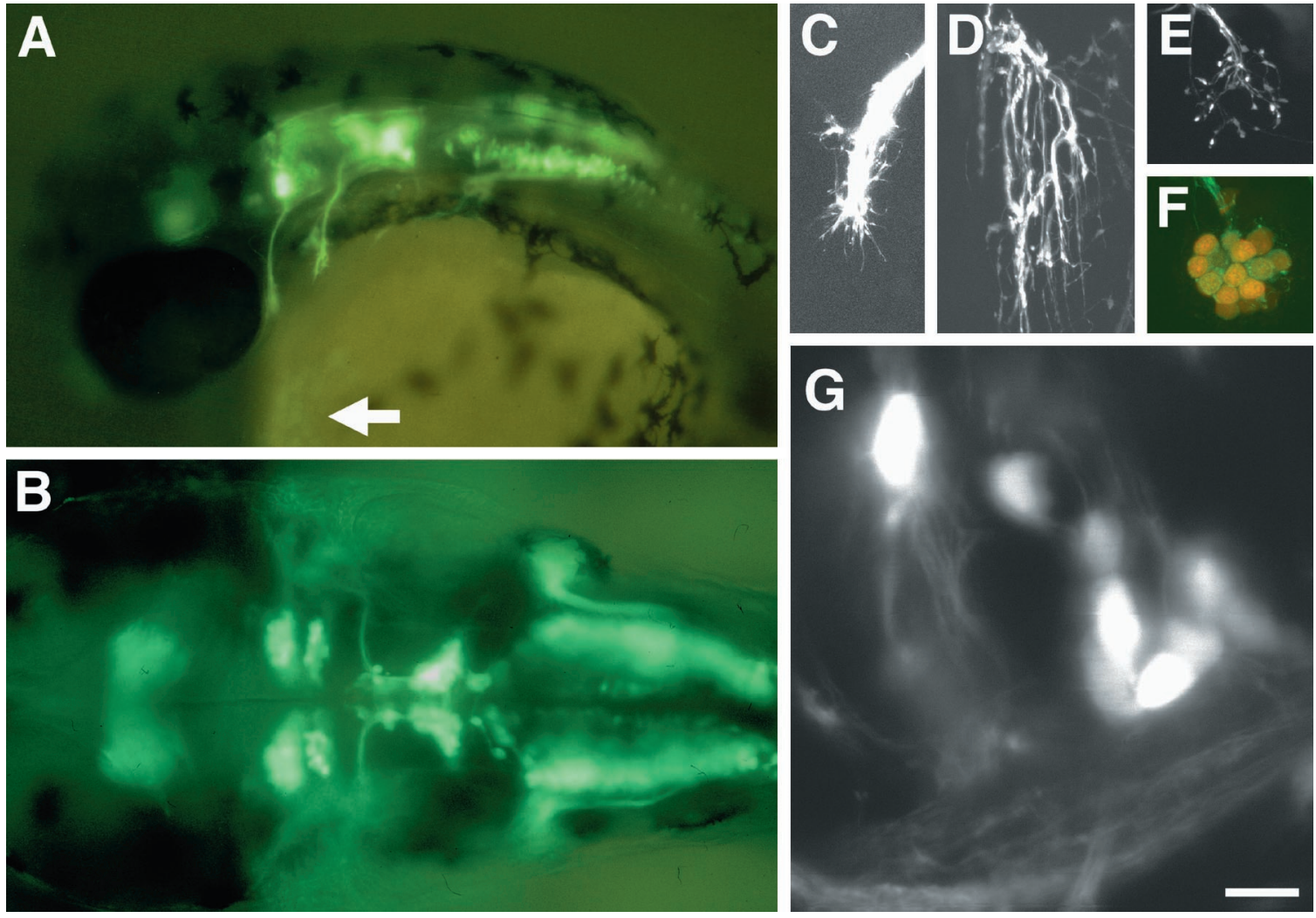

Figure 3. GFP expression in cranial motor neurons in the Isl1-GFP line. A, Lateral view of the head region of a 34 hr embryo. The arrow indicates GFP expression in the hatching gland cells. $B$, Dorsal view of the midbrain and the hindbrain of a $42 \mathrm{hr}$ embryo. In $A$ and $B$, pictures were taken under a conventional epifluorescence microscope. $C-G$, Close-up views of the GFP-positive nerve endings and neurons in the Isl1-GFP line using a confocal microscope. In panels $C-F$, composite pictures were generated from the stacked confocal images. $C$, Lateral view of the distal tips of the trigeminal motor axons at $30 \mathrm{hr}$. Note the fine filopodial structures extending from the distal ends of the axons. $D$, Lateral view of the neuromuscular junction in the levator arcus palatini (lap) muscle at $96 \mathrm{hr}$. Note the fine arborization of the nerve branches innervating individual muscle fibers. $E$, $F$, GFP expression in the efferent component of the lateral line nerves in a $96 \mathrm{hr}$ embryo. Close-up view of their endings at the neuromasts. The position of the neuromast corresponds to $h$ in Figure $7 C$. In $F$, the larva was treated with the vital dye DASPEI, which stains hair cells. DASPEI signals appear as orange. Note that the nerve endings appear to terminate within the hair cells. $G$, A confocal optical section showing the trigeminal motor neurons at 105 hr. Dorsal view with medial to the top. A cluster of neurons situated on the right correspond to those in the posterior (Vp) cluster of the trigeminal motor neurons (Figs. 5-7). Note that individual GFP-positive neurons are readily visualized. In all figures, anterior is to the left. In lateral views, dorsal is up. Scale bar: $A, 110 \mu \mathrm{m} ; B, 60 \mu \mathrm{m} ; C-F, 20 \mu \mathrm{m} ; G, 10 \mu \mathrm{m}$. 
Figure 4. Comparison of the expression patterns of Islet-1 (Isl1) and GFP mRNAs in the cranial motor and sensory neurons. $A, C, E, G F P$ mRNA expression in embryos from the Isl1-GFP line. $B, D, F$, Isll mRNA expression in wild-type embryos. $A, B$, Dorsal views of $28 \mathrm{hr}$ embryos. GFP and Isl 1 mRNAs are both expressed in presumptive cranial motor neurons. Whereas Isl1 mRNA is expressed in the trigeminal ganglion cells $(t g), G F P$ mRNA is not expressed in these cells. $C$, $D$, Dorsal views of $40 \mathrm{hr}$ embryos. Isll mRNA expression in the nIII and nIV neurons is difficult to see because many other neighboring cells also express Isl1 mRNA (D, asterisk). Arrows in $D$ indicate cells that do not express GFP mRNA in the Isl1-GFP line. The nVI and nIX neurons do not express GFP in the Isl1-GFP zebrafish (see the following figures). $E, F$, Lateral views of $40 \mathrm{hr}$ embryos. GFP is expressed in cells in the facial sensory ganglion $(f s)$, glossopharyngeal sensory ganglion $(g s)$, and vagus sensory ganglions ( $v s)$, but is not expressed in cells in the trigeminal sensory ganglion $(t g)$. Scale bar, $100 \mu \mathrm{m}$.

\section{GFP}
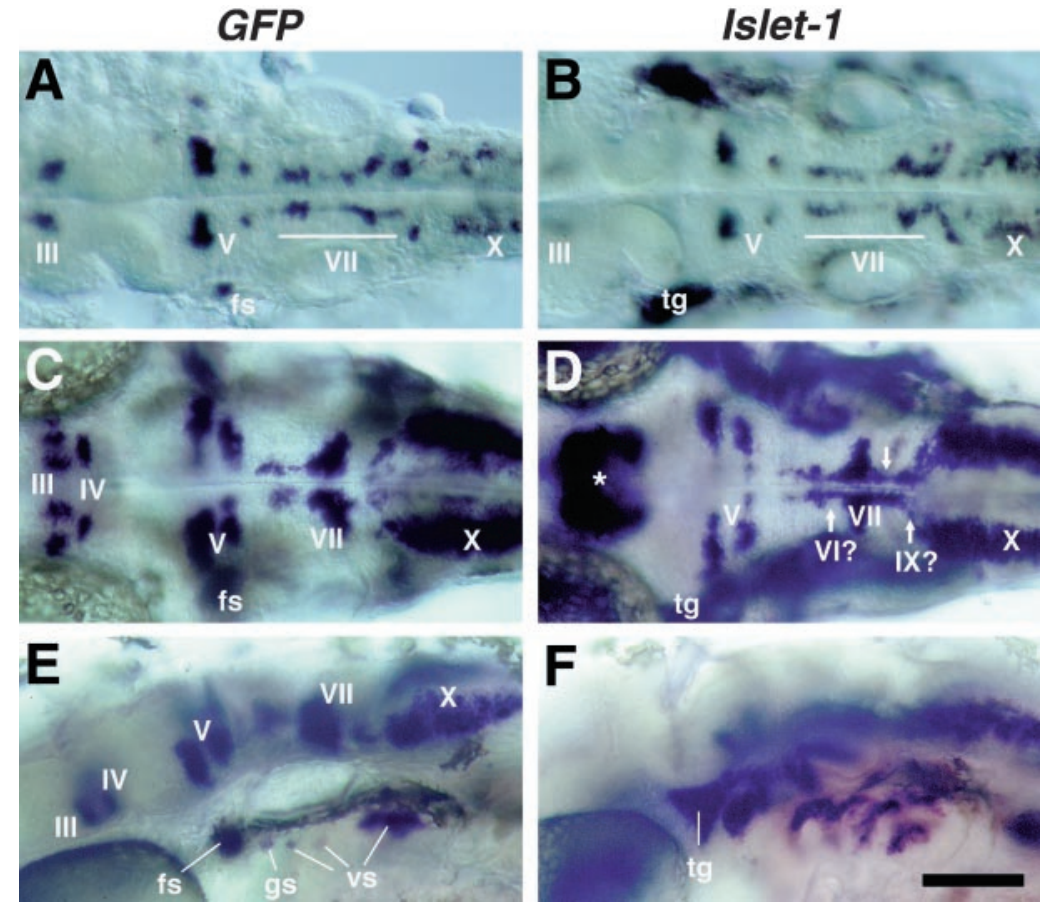

the constructs (Fig. 2A, arrowhead). Thus, hatching gland expression of GFP served as a positive control for the injections. At least 50 surviving embryos were examined for each construct. One construct, CMICP-GFP (Fig. 1D), which carries a $15 \mathrm{~kb}$ EcoRI fragment (Fig. 1A,CM), was found to drive GFP expression in branchiomotor neurons innervating the pharyngeal arches in injected embryos (Fig. 2A, arrows). Additionally, we identified another enhancer fragment (Fig. $1 A, S S$ ) that was capable of driving GFP expression in the trigeminal ganglion cells and Rohon-Beard cells in the spinal cord (Fig. 2B). Isll is normally expressed by both of these cell types as well as the motor neurons and the hatching gland (Inoue et al., 1994). All of the other EcoRI fragments were negative: we carefully looked for GFP expression in primary motor neurons in the spinal cord, but expression was not detected in these cells that normally express Isl1. Furthermore, Isl1 is expressed in many other cells such as those in the epiphysis and the nucleus of the posterior commissure, but no constructs gave GFP expression in these cells. It should be noted that because transgenes are mosaically expressed in the injected embryos, our negative results should not be construed as conclusive.

To establish stable transgenic zebrafish, CMICP-GFPinjected embryos were raised to sexual maturity, and either incrossed with each other or outcrossed with noninjected adult fish to generate F1 progeny. Of 113 fish, three produced embryos expressing GFP in a subset of Isl1-expressing cells, including the cranial motor neurons (Fig. 3A,B). The expression patterns of GFP in embryos derived from the three founder fish were identical, although expression levels varied. The line with the highest GFP expression, referred to as Isl1-GFP hereafter, was chosen for further analysis. In this study, we focused on GFP expression patterns of the Isl1-GFP line in the cranial region. As predicted by the transient expression analysis, GFP was also expressed in the hatching gland cells (Fig. $3 A$, arrow). Additionally, some secondary motor neurons in the spinal cord and a small number of interneurons in the CNS also expressed GFP (data not shown).

\section{The Isl1-GFP line enables clear visualization of cranial motor neurons}

To examine how faithfully GFP expression reflects the expression of the intrinsic Isl1 mRNA in the Isl1-GFP line, we compared the distribution of GFP mRNA and Isll mRNA. GFP mRNA (Fig. $4 A, C, E)$ is expressed in a majority of cranial motor neurons in a similar manner to Isl1 mRNA (Fig. 4B,D,F). Outside the CNS, $G F P$ mRNA is also expressed in all the branchiomeric sensory ganglion cells except for the trigeminal (V) ganglion cells (Fig. $4 E, f s, g s, v s)$. Branchiomeric nerves (V, VII, IX, and X) are nerves innervating derivatives of the pharyngeal arches and are known to include both motor and sensory components. Besides, GFP is also expressed in the efferent neurons for the lateral line and vestibuloacoustic nerves, as described in more detail later.

We examined GFP expression in live zebrafish most often with a confocal microscope (see Materials and Methods). After obtaining a series of optical sections, composite pictures were generated from the stacked images. The expression levels of GFP were high enough to visualize both fine neuronal processes during development such as filopodia (Fig. $3 C$ ) and fine terminal arbors of the motor axons (Fig. 3D), suggesting that neuronal processes were visualizable in their entirety in the Isl1-GFP line.

The expression of Isl1 in motor neurons begins early in their development. Likewise, GFP expression was detected early in cranial motor/efferent neurons (Fig. $5 A$, see GFP expression in a $21 \mathrm{hr}$ embryo). The transparent nature of zebrafish embryos and larvae enabled visualization of GFP-labeled neurons and their processes at high resolution in living animals. This was expected at early stages such as $24 \mathrm{hr}$ because the early hindbrain is relatively simple (Chandrasekhar et al., 1997), but was also the case at much later stages, such as day 4 when the hindbrain is much larger and much more complex, and the larvae begin complex behaviors such as swimming. An example is shown in Figure $3 G$ (an optical section) in which individual neurons in the trigeminal motor nucleus, which lie deep in the hindbrain, were 

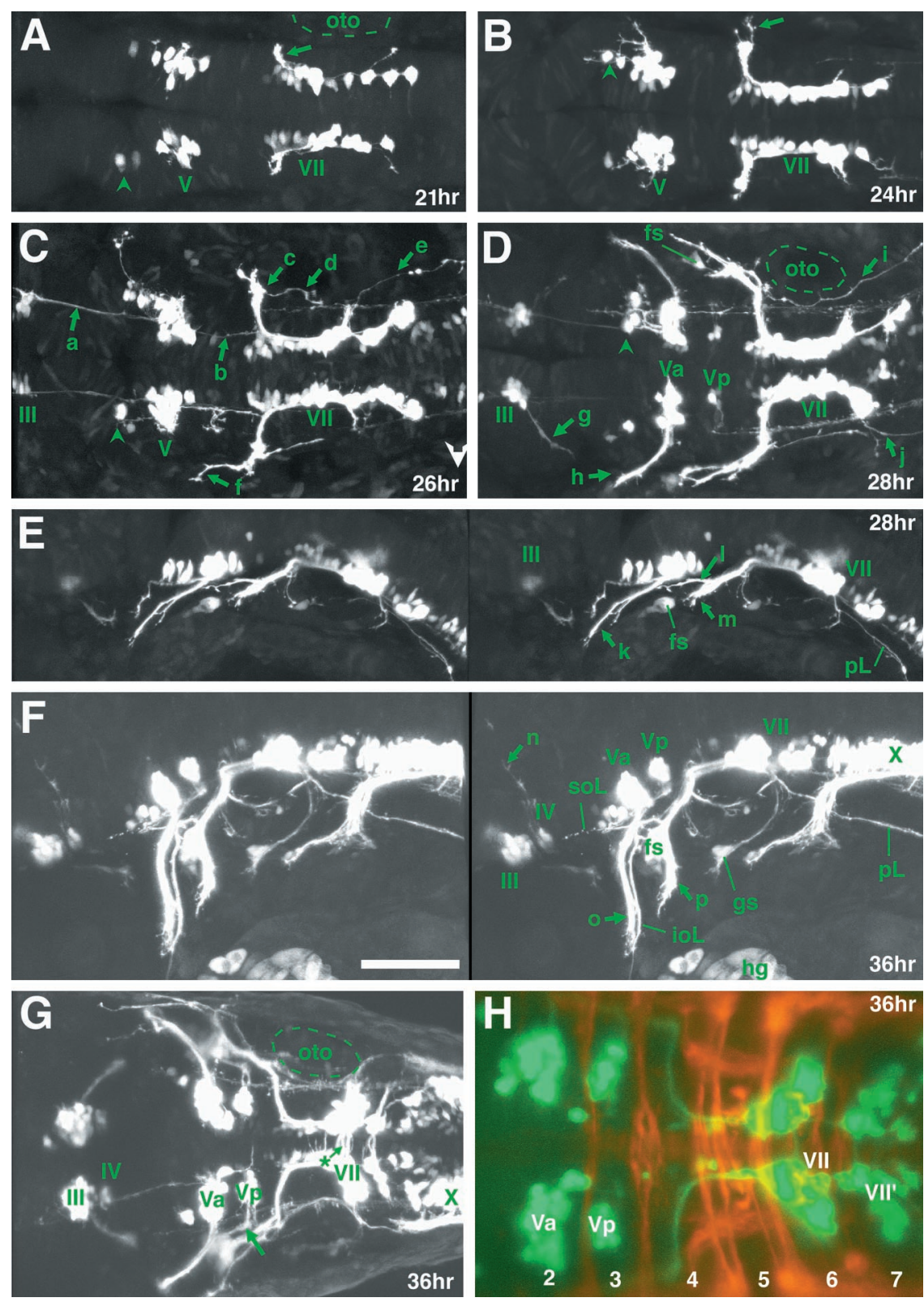

Figure 5. GFP expression early in the development of the cranial motor neurons $(21-36 \mathrm{hr}$ ) in the Isl1-GFP line. All micrographs are confocal composite images generated from a series of optical sections. $E$ and $F$ are stereographic images. $A$, Dorsal view of a $21 \mathrm{hr}$ embryo. The arrow indicates the nVII/OLe axons. At this stage most of the GFP-positive $\mathrm{nVII} / \mathrm{OLe}$ neurons are located in $\mathrm{r} 4$ and $\mathrm{r} 5$. Rhombomere boundaries were estimated by designating the hindbrain region spanning the central half of the otocyst (oto) as r5, and those spanning the anterior and posterior quarters of the otocyst as parts of $\mathrm{r} 4$ and r6, respectively. $B$, Dorsal view of a $24 \mathrm{hr}$ embryo. The pioneering axons from the $\mathrm{nVII} / \mathrm{OLe}$ neurons are seen outside the hindbrain (arrow). C, Dorsal view of a 26 hr embryo. *Longitudinal processes that connect the nIII, $\mathrm{nV}$, and $\mathrm{nVII}$ motor nuclei are visible (for example, $a$ and $b$ ). ${ }^{*}$ The significance of these early-forming axons is unknown. The majority of the nVII/OLe axons exit from the hindbrain $(c) . f$ indicates anteriorly projecting axons that subsequently extend into the lateral line system (corresponding to $l$ in $E$ ). D, Dorsal view of a $28 \mathrm{hr}$ embryo. $g$ indicates axons from the nIII neurons. $h$ indicates the peripherally extending axons from the Va cluster of the $\mathrm{nV}$ neurons. $d$ in $C$ and $i$ in $D$ indicate efferent axons extending into the posterior lateral line. *Efferent axons for the posterior lateral line also exit from the hindbrain via a posteriorly located exit point ( $e$ in $C$ and $j$ in $D$; Metcalfe et al., 1985). *Arrowheads in $A-D$ indicate GFP-positive cells located anteriorly to the main $\mathrm{nV}$ cluster. * It is not clear whether these cells constitute a part of the $\mathrm{nV}$ neurons. $E$, Lateral view of a $28 \mathrm{hr}$ embryo. $k$ indicates the peripherally extending axons from the $\mathrm{nV}$ neurons. $l$ indicates diverging axons extending into the lateral line system, whereas $m$ indicates the main nVII/OLe axons (mostly, the facial motor axons). It should be noted that, viewed laterally, the efferent axons for the posterior lateral line appear interrupted by the otocyst because of the opaque nature of the otolith (also in Figs. $5 F$, $6 B, E, 7 C) . F$, Lateral view of a $36 \mathrm{hr}$ embryo. $n$ indicates dorsally extending axons from the nIV neurons. $o$ and $p$ indicate the peripherally extending axons from the $\mathrm{nV}$ and $\mathrm{nVII} /$ OLe neurons, respectively. $G$, Dorsal view of a $36 \mathrm{hr}$ embryo. The thick arrow indicates axons from the $\mathrm{Vp}$ cluster of the $\mathrm{nV}$ neurons. *Contralaterally projecting neurites from the nVII/ OLe neurons are visible (asterisk). $H$, A confocal composite image of a $36 \mathrm{hr}$ embryo in which hindbrain commissural axons that are located at the rhombomere boundary are labeled with the zn5 antibody. Dorsal view. Numbers listed are rhombomere num-

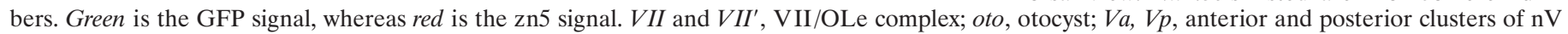

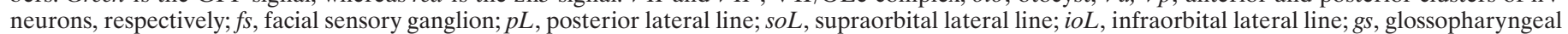

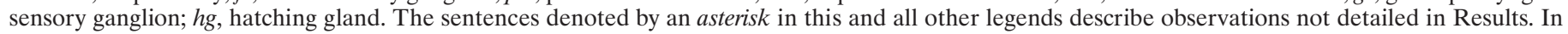
lateral views, dorsal is to the top. Scale bar, $A-G, 100 \mu \mathrm{m} ; H, 50 \mu \mathrm{m}$.

visualized even in a $4 \mathrm{~d}$, live larva. Thus, it is possible to observe development of the cranial motor neurons in intact animals with a high level of resolution from early stages to relatively mature stages in the Isl1-GFP line.

\section{Overall development of the trigeminal motor (nV) neurons}

Although axonal outgrowth by the $\mathrm{nV}$ neurons in zebrafish has been examined by a variety of methods, including retrograde labeling or immunohistochemistry (Hatta et al., 1990; Chandrasekhar et al., 1997; Schilling and Kimmel, 1997), their overall development is still largely obscure because of the limitation of these methods. Given below is a description of the overall development of the $\mathrm{nV}$ neurons as gleaned from observations of the
Isl1-GFP line. Some of the figures (Figs. 5-7) present micrographs in stereographic pairs.

GFP is expressed in the $\mathrm{nV}$ neurons, but not in the trigeminal sensory ganglion cells (Isl1 is expressed in both; compare Fig. $4 A, C, E$ with $B, D, F)$. Thus, all the GFP-labeled axons in cranial nerve $\mathrm{V}$ are motor axons. GFP-positive $\mathrm{nV}$ neurons are evident as early as $21 \mathrm{hr}$ (Fig. 5A) and progressively increase in number with further development (Fig. $5 A-C$ ). Thus, GFP is expressed early enough so that the entire time course of axonal outgrowth by the $\mathrm{nV}$ neurons could be observed. By $28 \mathrm{hr}$, the motor axons derived from the $\mathrm{nV}$ neurons (Fig. $5 D, V a$ ) have extended laterally to exit from the hindbrain into the periphery (Fig. $5 D, h, E, k$ ). At about the same time, an additional more posterior cluster of $\mathrm{nV}$ neurons begins to express GFP (Fig. 5D, $V p$ ). GFP-positive neurons in the 
Figure 6. GFP expression in the Isl1-GFP line on days 2 and 3 of development. All panels except $C$ are confocal stereographic images. $A$, Dorsal view of a $48 \mathrm{hr}$ embryo. The asterisk indicates medially located $\mathrm{nV}$ neurons that are presumably born later than laterally located $\mathrm{nV}$ neurons. $B$, Lateral view of a $48 \mathrm{hr}$ embryo. $C$, Schematic illustration of GFP-labeled nerves in B. ipsi and contra indicate fourth nerves from the nIV neurons on the ipsilateral and the contralateral sides, respectively. $a$ and $b$ indicate the peripherally projecting axons from the facial sensory ganglion cells. The axons of $a$ take an internal pathway, whereas those of $b$ take an external pathway. The axons indicated by $a$ and $b$, respectively, correspond to those indicated by in and ex in Figure $9 A-C$. Thin arrows show GFP-expressing cells that presumably do not correspond to motor neurons. The thick arrow indicates an OLe nerve branch extending into the hair cells in the otocyst. The asterisk indicates branching processes of the OLe nerves, which are added later to the major branches. The arrowhead indicates the distal tip of the fifth motor axons. $D$, Ventral view of a $62 \mathrm{hr}$ embryo. $E$, Lateral view of a $72 \mathrm{hr}$ larva. The embryo in $D$ and the larva in $E$ were treated with the vital dye DASPEI, which stains hair cells in lateral line neuromasts. DASPEI signals appear as yellow. $I, D$, and $P$ indicate the intermediate, distal, and proximal branches of the fifth motor nerve, respectively. Arrows in $D$ and $E$ indicate peripherally projecting axons from the facial sensory ganglion cells (equivalent to $a$ and $b$ in $C$ ). Among them, in indicates the axons that are distal tip of the axons indicated as $a$ in $C$. They take an internal pathway. Most part of their tract is out-of-focus. The arrowhead indicates the junction of the four motor nerves (both sides of the fifth and seventh motor nerves). $c$ and $d$ indicate nerve branches that innervate the intermandibularis anterior and posterior (ima and imp), and the hyohyal $(h h)$, respectively (Fig. $7 E$ ). $e$ indicates the neuromuscular junction at the levator arcus palatini (lap), whereas $f$ indicates the neuromuscular junctions at the abductor hyomandibulae $(a h)$ and the abductor operculi (ao) (Fig. 7D). $g$ indicates distal branches of OLe nerves from the main nVII/OLe nerve. Asterisks indicate nerve endings of the OLe system in the ear (Fig. $7 C$ ). ${ }^{*} c n$ is likely to be the ciliary nerve innervating the lens muscle for visual accommodation. *The lens muscle in teleost was shown to be controlled by the postganglionic fibers of the oculomotor (parasympathetic) nerve (Somiya, 1987), and thus, GFP should be expressed in the parasympathetic ganglion cells corresponding to the ciliary nerve. $a-f s$, centrally projecting afferent facial sensory axons; $p L$, posterior lateral line; $v s$, vagus sensory ganglion. Scale bar, $100 \mu \mathrm{m}$.
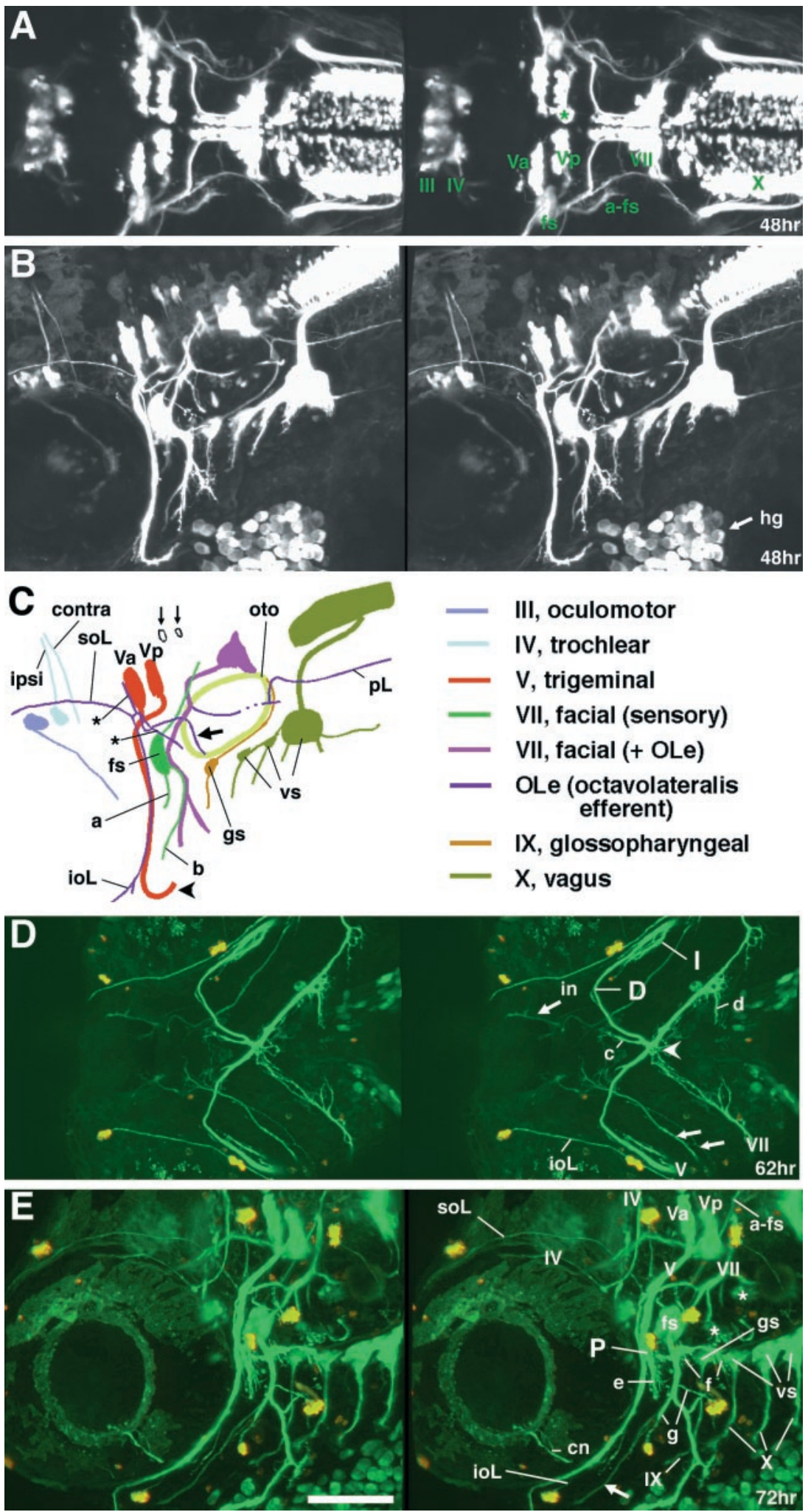

Vp cluster increase in number as development proceeds. At $\sim 36$ hr, their axons (Fig. 5G, thick arrow) have extended laterally and then anteriorly to merge with the axons from the Va cluster. Our observations showed that, throughout development, the $\mathrm{Va}$ and Vp clusters lie discretely apart, and that their axons follow their own pathways within the hindbrain until joining together at a common hindbrain exit point (Figs. $5 F, G, 6 A-C, E, 7 A, 8 C, F, I$ ).

We examined the locations of the $\mathrm{Va}$ and $\mathrm{Vp}$ clusters with respect to the hindbrain rhombomeres using zn5 antibody, which labels hindbrain commissural axons that are located at the rhom- 

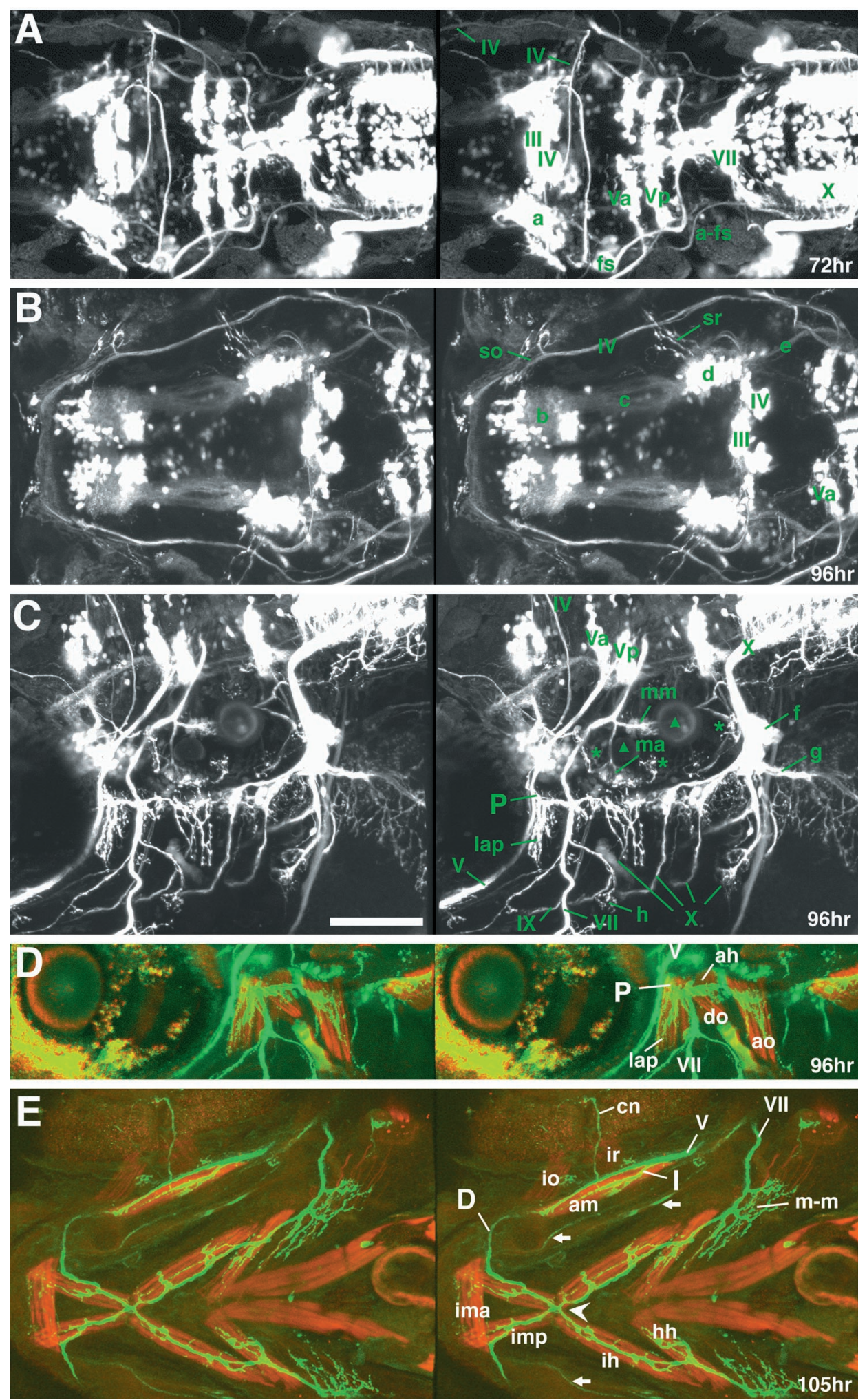

Figure 7. GFP expression in the Isl1-GFP line on days 3 and 4 of development. All figures are stereographic pictures reconstructed from the stacked confocal images. $A$, Dorsal view of a $72 \mathrm{hr}$ larva. $B$, Dorsal view of a $96 \mathrm{hr}$ larva. GFP is also expressed in cells other than motor neurons in the brain. The identities of these cells are not known. They are a cluster of cells in the telencephalon $(b)$ and a cluster of cells in the mesencephalic region $(d)$. Tracts are formed between these two clusters $(c)$. The cluster of the mesencephalic region $(d)$ also sends processes posteriorly ( $e$ ). $a$ in $A$ corresponds to $d$ in $B$. For these cells and tracts, see also the legend to Figure $9 E$. C, Lateral view of a $96 \mathrm{hr}$ larva. Five terminal-like structures are observed in the ear. These appear to correspond to the anterior macula ( $m a$ ) accompanied by the anterior otolith (the anteriorly located triangle), the medial macula $(\mathrm{mm})$ accompanied by the posterior otolith (the posteriorly located triangle), and the three crista ampullarises (asterisks) associated with the anterior, lateral, and posterior canals. Nomenclatures are according to Whitfield et al. (1996). $f$ indicates the largest vagus sensory ganglion, which is likely to include neurons innervating visceral organs. $g$ indicates the vagus nerve extending into the visceral organs. The nerve is likely to contain sensory components and visceral motor (parasympathetic) components. $h$ indicates the endings of the OLe nerve at a lateral line neuromast. Higher magnification views of the corresponding structures are shown in Figure $3, E$ and $F$. In $B$ and $C$, elaborated fine branches are visible at neuromuscular junctions (for example, so and $s r$ in $B$, lap in $C) . D, E$, Fixed larvae were treated with rhodamine-phalloidin to reveal actin filaments of muscles. Green is the GFP signal, whereas red is the rhodamine-phalloidin signal. $D$, Lateral view of a $96 \mathrm{hr}$ larva. $E$, Ventral view of a $105 \mathrm{hr}$ larva. $P, I$, and $D$ in $C-E$ indicate the proximal, intermediate, and distal branches of the fifth motor nerve, respectively. The proximal branch of the fifth nerve innervates the lap and do muscles, the intermediate branch innervates the am muscle, and the distal branch innervates the ima and imp muscles. The configuration is schematically summarized in Figure 8 A. Arrows indicate peripherally projecting axons from the facial sensory ganglion cells. *The seventh motor nerves innervate surfacial membranous muscles (indicated as $m-m$ ), which presumably corresponds to the platysma in higher vertebrates. The arrowhead indicates the junction of the four motor nerves (both sides of the fifth and seventh motor nerves). Abbreviations for muscles (so, sr, io, ir, lap, do, am, ima, imp, ah, ao, ih, and $h h$ ) are shown in Table $1 .{ }^{*} \mathrm{c} n$ is likely to be the ciliary nerve (Fig. 6E, legend). Scale bar, $100 \mu \mathrm{m}$. bomere boundaries (Trevarrow et al., 1990). Figure $5 H$ shows that the $\mathrm{Va}$ and $\mathrm{Vp}$ lie in $\mathrm{r} 2$ and $\mathrm{r} 3$, respectively. The hindbrain exit point of the fifth motor nerve lies in $\mathrm{r} 2$ (Fig. $5 G$ ). The locations and distributions of the $\mathrm{nV}$ neurons are, in general, consistent with those revealed by retrograde labeling with DiI (Chandrasekhar et al., 1997). However, the number of neurons that are labeled at a given stage is much larger in our analysis. Presum- ably, this is attributable to the fact that DiI backlabeling from the target pharyngeal arch only labels those neurons whose axons have reached the arch and that backlabeling may be incomplete, whereas in the Isl1-GFP line, all the motor neurons, even though they have not reached their targets, are labeled. Furthermore, we found that cells in a medial position were labeled in our Isl1-GFP line (Fig. 6A, asterisk), whereas at similar stages backlabeling with 

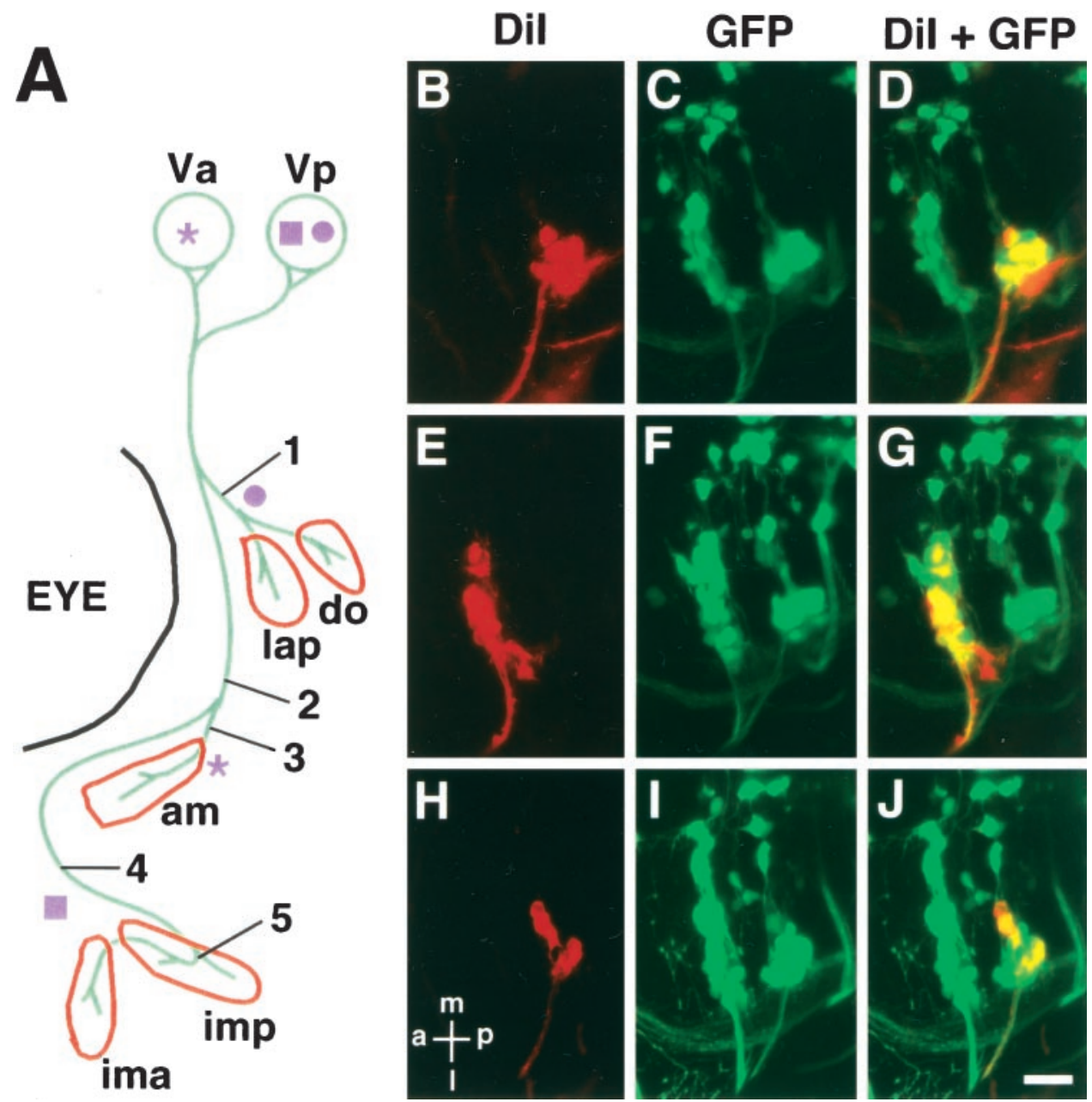

Figure 8. Position of the trigeminal motor $(\mathrm{nV})$ neurons with respect to the peripheral nerve branches. $A$, A diagram showing dye application sites and a summary of the results. Trigeminal motor neurons were retrogradely labeled with DiI. DiI application was performed at positions $1-5$. The results showed that Va solely consists of neurons projecting to the intermediate branch (asterisk), whereas $\mathrm{Vp}$ consists of neurons projecting to the either proximal (circle) or distal branch (square). Abbreviations for the muscles are the same as those shown in Table 1. In $B-J$, all pictures are dorsal views (composite pictures made from stacked confocal images) of larvae at $\sim 90 \mathrm{hr}$ with anterior to the left and medial to the top. $B-D$, DiI was applied at position 1 . Neurons belonging to the posterior cluster $(V p)$ of the $n V$ neurons were labeled with DiI. $E-G$, DiI was applied at position 3. Neurons belonging to the anterior cluster (Va) were labeled with DiI. $H-J$, DiI was applied at position 4. Neurons belonging to the posterior cluster (Vp) were labeled. Scale bar, $20 \mu \mathrm{m}$.
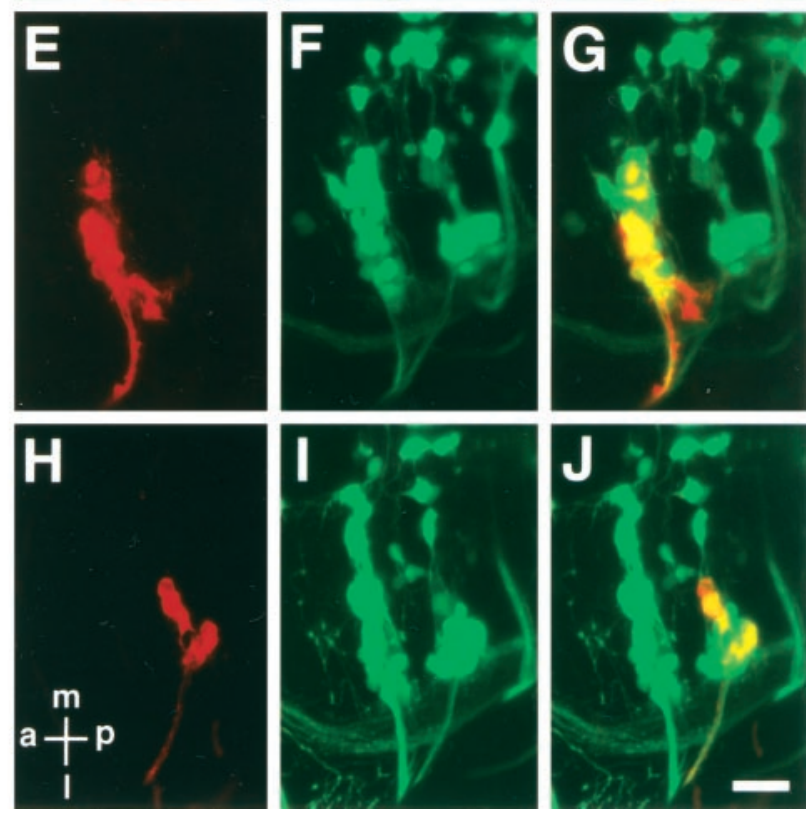

DiI only labeled cells in a lateral position (Chandrasekhar et al., 1997). Thus, it could be that the earliest $\mathrm{nV}$ neurons are born medially and migrate laterally. This is consistent with the possibility that $\mathrm{nV}$ neurons are arranged medial to lateral within the hindbrain according to the time they are born and differentiate with early ones laterally and later ones more medially. A similar pattern of development of trigeminal motor neurons has also been described in the chick embryo (Heaton and Moody, 1980; Simon et al., 1994).

Once outside of the hindbrain, axons of the $\mathrm{nV}$ neurons extend ventrally along the posterior edge of the eye (Fig. $5 F, o$ ), and by $48 \mathrm{hr}$ the axons turn posteriorly (Fig. 6C, arrowhead). At 62 and $72 \mathrm{hr}$, nerve branches innervating the mandibular arch muscles are clearly visible ( $I, D$ in Fig. $6 D ; P$ in Fig. $6 E$ ). Among them, the distal branch of the fifth nerve ( $D$ in Fig. $6 D)$ runs in the posteromedial direction to the midline, where it merges with both the contralateral fifth nerve and the distal tip of the seventh (facial) nerve (Figs. 6D, 7E, arrowhead). As the zebrafish developed, the labeled fifth axons progressively shifted anteriorly because of extension of the jaw (compare Fig. $6 D$ with $7 E$ ).

Correspondence between peripheral branches of the fifth nerve and target muscles was determined by labeling of fixed sample with rhodamine-phalloidin to show the configuration of the motor nerves and their target mandibular muscles (Fig. 7D,E; see Table 1 for summary of the nomenclature of the mandibular arch muscles and the extraocular muscles, the abbreviated forms of

their names, and their innervation patterns). The proximal branch of the fifth nerve ( $P$ in Figs. $6 E, 7 C, D)$ innervates the levator arcus palatini (lap) and dilator operculi (do) muscles. The intermediate branch ( $I$ in Figs. $6 D, 7 E$ ) innervates the abductor mandibulae $(\mathrm{am})$ muscle. Finally, the distal branch ( $D$ in Figs. $6 D, 7 E)$ innervates the intermandibularis anterior and posterior (ima and imp) muscles. This configuration is schematically shown in Figure $8 A$.

\section{Trigeminal motor axons from different rhombomeres innervate distinct target muscles}

Because the trigeminal motor neurons innervate several different muscles and they are organized into two clusters, Va in $\mathrm{r} 2$ and Vp in $r 3$, we wondered whether the two clusters of trigeminal motor neurons innervated different target muscles. To examine this question, we took advantage of the fact that $\mathrm{nV}$ nerve and branches are readily visible in the Isl1-GFP line. We selectively backlabeled those motor neurons that supply axons to a specific nerve branch by application of DiI to that branch. In the periphery, there are three major branches, and DiI was applied at five different positions along the branches in 72-90 hr larvae (Fig. 8A, 1-5). DiI application at position 1 , which contains axons innervating the lap and $d o$ muscles, labeled Vp neurons (Fig. $8 B-D$; $n=5)$. DiI application at position 2, which contains axons innervating the $a m$, ima, and imp muscles, labeled $\mathrm{Va}$ and $\mathrm{Vp}$ neurons (data not shown; $n=2$ ). Application of DiI at position 3, 


\begin{tabular}{|c|c|c|}
\hline Region and muscles & Abbreviation & Innervation \\
\hline \multicolumn{3}{|l|}{ Extraocular } \\
\hline Superior oblique & so & IV \\
\hline Inferior oblique $^{a}$ & io $^{a}$ & III \\
\hline Superior rectus & sr & III \\
\hline Inferior rectus & ir & III \\
\hline Medial rectus & $\mathrm{mr}$ & III \\
\hline Lateral (posterior) rectus ${ }^{b}$ & $\operatorname{lr}^{b}$ & VI \\
\hline \multicolumn{3}{|l|}{ Mandibular arch } \\
\hline Intermandibularis anterior & ima & V \\
\hline Intermandibularis posterior & imp & V \\
\hline Abductor mandibulae & $\mathrm{am}$ & V \\
\hline Levator arcus palatini & lap & $\mathrm{V}$ \\
\hline Dilator operculi & do & $\mathrm{V}$ \\
\hline \multicolumn{3}{|l|}{ Hyoid arch } \\
\hline Interhyal & ih & VII \\
\hline Hyohyal & hh & V II \\
\hline Abductor hyomandibulae & ah & VII \\
\hline Abductor operculi & ao & VII \\
\hline
\end{tabular}

Nomenclatures are according to Schilling and Kimmel (1997).

${ }^{a}$ The inferior oblique (io) muscle, which is normally innervated by the third nerve, appears not to be innervated by the GFP-labeled fibers between 72 and $96 \mathrm{hr}$ in the Isl1-GFP line, suggesting that, among nIII neurons, those supplying the io do not express GFP. The rest of three muscle groups ( $\mathrm{mr}$, sr, and ir) are innervated by the GFP-labeled fibers by $72 \mathrm{hr}$.

${ }^{b} \mathrm{GFP}$ is not expressed in the abducens motor (nVI) neurons in the Isl1-GFP line. Consequently, the lateral rectus (lr) muscle is not innervated by the GFP-labeled fibers.

which contains axons innervating the am muscle, labeled $\mathrm{Va}$ neurons (Fig. $8 E-G ; n=6$ ). Finally, DiI application at position 4 or 5, which contains axons innervating the ima and imp muscles, labeled Vp neurons (Fig. $8 H-J ; n=7$ for 4 and $n=4$ for 5). These results indicate that the $\mathrm{Va}$ neurons exclusively innervate the am muscle, whereas the Vp neurons innervate the lap, do, ima, and imp muscles. Thus, the two clusters of trigeminal motor neurons innervate distinct target muscles, and this can be seen at early larval stages. These results confirm the pattern of innervation found in adult fish (Song and Boord, 1993). Because Va and $\mathrm{Vp}$ are located in different rhombomeres and appear not to intermingle during development, our results are consistent with the notion that $\mathrm{nV}$ neurons born in $\mathrm{r} 2$ and $\mathrm{r} 3$ adopt distinct fates, and this suggests that the segment of origin determines the identity of the $\mathrm{nV}$ neurons.

\section{Characterization of GFP-positive cranial nerves other than the trigeminal nerve}

Below we describe the GFP-positive cranial motor and sensory neurons because many of them have not yet been fully characterized in developing zebrafish. To keep the text concise, some minor observations are described only in the figure legends (Figs. $5-7$, see the comments denoted by asterisks). Some of our findings overlap with previously reported results (Chandrasekhar et al., 1997).

\section{Facial (VII) and octavolateralis efferent (OLe) nerves}

In addition to motor neurons, GFP is expressed in the efferent neurons for the lateral line and the vestibuloacoustic nerves. The lateral line, which is unique to aquatic vertebrates, is a sensory system mainly responsible for detection of water displacement (Coombs et al., 1989). The lateral line nerve as well as the vestibulo-acoustic nerve terminates at hair cells that are innervated by both sensory and efferent (octavolateralis efferent, OLe) nerves (Roberts and Meredith, 1989; 1992; Highstein, 1991). The facial motor (nVII) and OLe neurons are located in close proximity, and the efferent axons from both cell types extend together in the hindbrain. Therefore, we have combined the description of axonal outgrowth by both sets of efferent neurons.

GFP-positive nVII/OLe neurons (Fig. 5A, VII) and their axons (Fig. $5 A$, arrow) are evident by $21 \mathrm{hr}$. At $\sim 24 \mathrm{hr}$, the pioneering axons among the $\mathrm{nVII} / \mathrm{OLe}$ axons exit from the hindbrain (Fig. $5 B$, arrow), and by $26 \mathrm{hr}$, most of the $\mathrm{nVII} / \mathrm{OLe}$ axons have left the hindbrain (Fig. $5 C, c$ ). The thick, main bundle of nVII/ OLe axons (Fig. $5 E, m$ ) grows toward the facial ganglion (Fig. 5E, $f s$, which becomes discernible at $\sim 27-28 \mathrm{hr}$. By the facial ganglion, the main bundle grows ventrally (Fig. $5 F, p$ ). At $48 \mathrm{hr}$, the main bundle (Fig. $6 A-C$, purple) is mainly composed of the facial motor axons (see below for the sensory and OLe fibers). Projection of motor nerves to target muscles was confirmed by labeling muscles with rhodamine-phalloidin at later stages (Fig. $7 D, E$ ). The labeled seventh motor nerve innervates the $a h, a o, i h$, and $h h$ muscles (see Table 1 for abbreviations for the names of the muscles).

The nVII/OLe motor neurons appear to migrate caudally during development. At early stages $(21 \mathrm{hr})$, most of the nVII/ OLe neurons are localized in $\mathrm{r} 4$ and $\mathrm{r} 5$, as judged by comparison with the location of the otocyst (Fig. $5 A$, see its legend for assignment of rhombomere). In the next $15 \mathrm{hr}$, the nVII motor neurons were seen to migrate caudally such that by $36 \mathrm{hr}$ most of the motor neurons (Fig. 5H, VII, VII') were located in r6 with some in r7. Our findings confirm the hypothesis that the nVII/ OLe motor neurons migrate caudally, as first suggested by Chandrasekhar et al. (1997) based on a caudal shift of cells expressing the Isl1 protein and tag1 mRNA (cranial motor neuron markers) from $\mathrm{r} 4$ and $\mathrm{r} 5$ to $\mathrm{r} 6$ and $\mathrm{r} 7$. In this earlier study, however, there remained the possibility that the apparent caudal shift of the Isl1/tag1-positive cells was not caused by migration of neurons, but was caused by changes in gene expression by neurons in these rhombomeres. Because the nVII/OLe neurons (identified by their axonal trajectories) were seen to shift caudally in Isl1-GFP embryos by observing them at several different time points, it is clear that these neurons indeed do migrate caudally.

To get a clearer description of axonal outgrowth by the motor and sensory components, we made mosaic embryos, by isochronically transplanting cells of the Isl1-GFP line into wild-type embryos at the sphere stage. In the resultant embryos, GFP was expressed by only a small number of cranial motor and sensory neurons, making it possible to unambiguously follow axonal outgrowth in these cells. Interestingly, observations of facial sensory neurons in mosaic embryos showed that their centrally projecting axons ( $a$ - $f s$ in Figs. $6 A, E, 7 A, 9 A, C, D$; green in Fig. $6 C$ ) take a distinct pathway from the outgrowing $\mathrm{nVII} / \mathrm{OLe}$ motor axons. In amniotes, the motor/efferent axons and the centrally projecting sensory axons follow the same pathway. The centrally projecting axons initially extend dorsoposteriorly, turn medially into the hindbrain, and then posteriorly within the hindbrain (Figs. $7 A$, $a-f s ; 9 C, D)$ to terminate in the dorsoposterior part of the hindbrain by day 3 (Fig. $9 C, D$, arrowheads). The peripherally extending axons of the sensory nVII neurons diverge into two nerves as they extend in a ventroanterior direction (Fig. $6 C, a, b$ ), but they all eventually terminate around the mouth (Figs. $6 D, E, 7 E$, arrows; $9 A, B)$. This outgrowth pattern is consistent with the notion that the facial sensory nerves in vertebrates mostly convey gusta- 
Figure 9. Mosaic analysis of the Isl1-GFP line. Mosaic animals were made by isochronically transplanting cells of the Isl1-GFP line into the normal embryo at the sphere stage to obtain stochastic labeling of cranial motor and sensory neurons. All images except for $C$ are taken from larvae at $\sim 75 \mathrm{hr}$. $C$ is from a larva at $\sim 90$ hr. All figures are composite pictures made from the stacked confocal images. $A$, Lateral (slightly ventral) view of a mosaic larva in which GFP is expressed in the facial sensory ganglion cells $(f s) . B, C$, Ventral $(B)$ and dorsal (C) views of the same larva shown in $A$. $a-f s$ shows the centrally projecting afferent facial sensory axons, which terminate in the dorsoposterior part of the hindbrain (arrowhead). The asterisk indicates signals derived from GFP-expressing cells in the mesencephalic region (Fig. $7 A$, $a$; $B$, $d$; see also single and double asterisks in $E$ ). Peripherally extending axons from the facial sensory ganglion cells take internal (in) and external (ex) pathways. Axons of both internal and external pathways terminate near the mouth. The internal fibers (correspond to $a$ in Fig. $6 C$ ) take a deep route, and most of the parts of the fibers are not visible, partly because they run out of the focal plane, and partly because of the opaque nature of the eye (lateral view) and cartilages (ventral view). The external fibers initially take the same superficial pathway as the seventh motor nerve and then diverge (Fig. $6 C, b ; D, E, 7 E$, arrows).
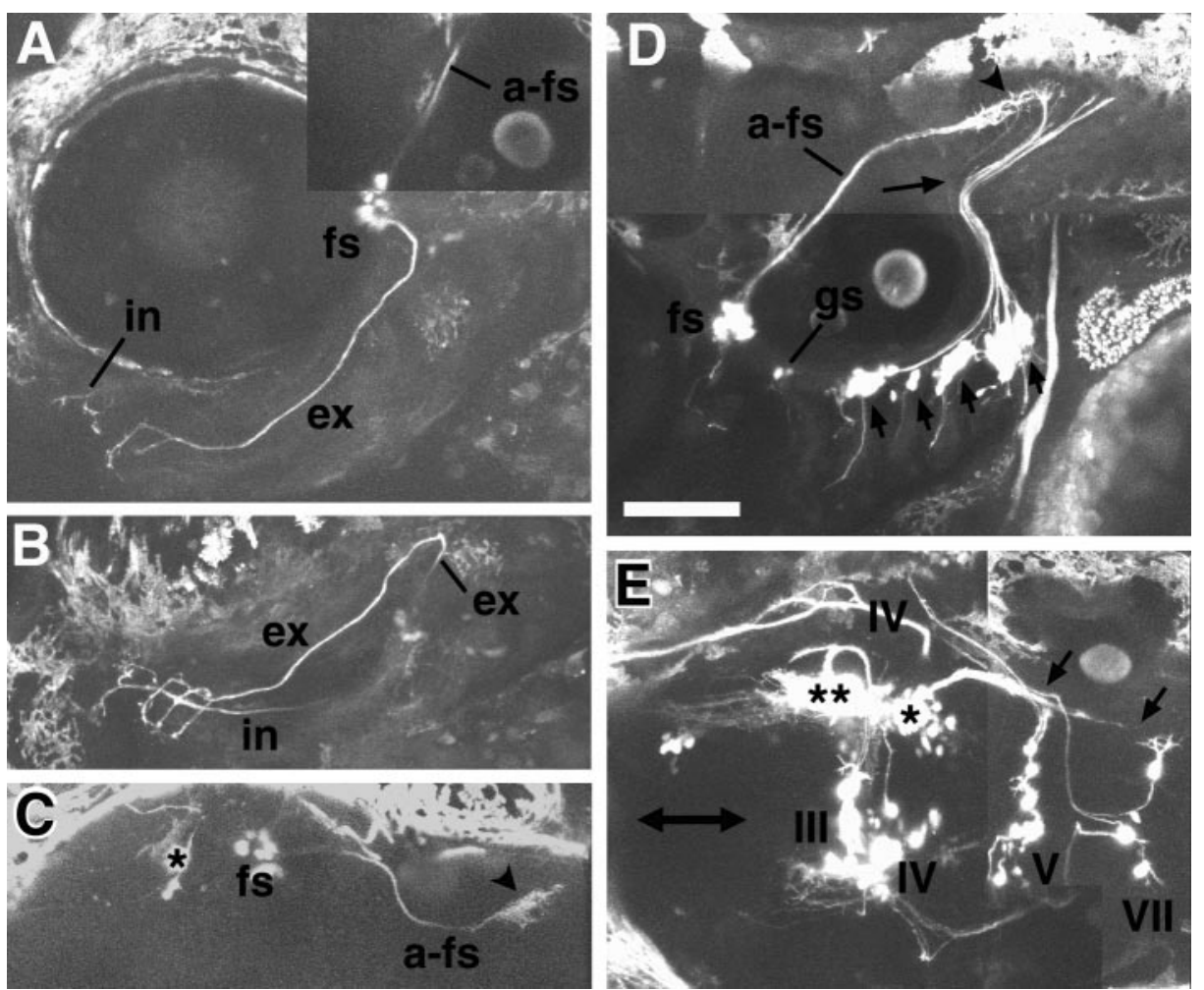

$D$, Lateral view of a mosaic larva in which GFP

is expressed in neurons in the facial sensory ganglion $(f s)$, in the glossopharyngeal sensory ganglion ( $g s)$, and the vagus sensory ganglion (short arrows). $a-f s$ shows the centrally projecting afferent facial sensory axons, which terminate in the dorsoposterior part of the hindbrain (arrowhead). The long thin arrow indicates the faintly fluorescent centrally projecting axons from the glossopharyngeal sensory neurons. Terminals of the centrally projecting axons from the facial, glossopharyngeal, and vagus ganglion cells are located near one another in the dorsoposterior part of the hindbrain. The terminal region is likely to correspond to the nucleus tractus solitarii in the adult fish. We have examined a number of mosaic embryos in which GFP was expressed in the facial sensory neurons $(n>15)$. In all cases, centrally projecting fibers took the same pathway (Figs. $6 A, E, 7 A, a$-fs; $6 C$, green), and peripherally projecting fibers eventually terminated around the mouth. $E$, Dorsal view of a mosaic embryo in which GFP is expressed in a number of cells other than the motor neurons in the brain. The double-headed arrow marks the midline. The double asterisks and the single asterisk indicate the GFP-positive cells in the mesencephalic region (Fig. $7 A, a ; B, d$ ). Anteriorly projecting processes from these cells (probably from the cells marked by the double asterisk) correspond to the tracts marked by $c$ in Figure $7 B$, whereas posteriorly projecting processes from these cells (probably from the cells marked by the single asterisk) correspond to the tracts marked by $e$ in Figure $7 B$. The posteriorly projecting axons may terminate around the trigeminal motor nucleus and facial motor nucleus (arrows). Scale bar, $100 \mu \mathrm{m}$.

tory information (Fig. $9 A-C$, see the legend to for further details on the peripheral trajectories).

Because GFP is not expressed in the sensory neurons related to the octavolateralis system, all of the GFP-labeled octavolateralis nerves in the periphery are efferent axons. Thus, it was possible to systematically follow the axonal outgrowth of the lateral line efferents. As described earlier, the pioneering axons among the $\mathrm{nVII} / \mathrm{OLe}$ axons exit from the hindbrain at $\sim 24 \mathrm{hr}$ (Fig. $5 B$, arrow). In vivo, time-lapse microscopic observations of these embryos suggested that these pioneering axons correspond to the OLe axons (data not shown). These axons extend both anteriorly (Fig. 5C, $f$ ) and posteriorly (Fig. $5 C, d ; D, i$ ) from the exit point. Posteriorly extending axons are likely to project to the posterior lateral line (Metcalfe et al., 1985). The anteriorly extending nerve takes a superficial pathway (Fig. $5 E, l$ ), and subsequently, branches with one branch extending anteriorly [supraorbital lateral line (soL)] and the other extending ventrally [infraorbital lateral line (ioL)] (Figs. 5F, 6B, C). Later in development, other branching processes can also be recognized (Fig. 6C, asterisks; $E$, $g$ ). Vital staining of hair cells in the lateral line neuromasts by DASPEI revealed that the OLe axons were found near the neuromasts as expected (Fig. 6D,E). Their endings spread into fine branches terminating in the circumference of the individual hair cells (Fig. $3 E, F$ ). Branches of the OLe nerves were also seen to project to the hair cells in the otocyst (Fig. 7C, $m m, m a$, asterisks). Thus, OLe nerves project to hair cells in the lateral line and otocyst quite early in their development, suggesting that hair cells receive efferent input from the onset of their function.

\section{Other cranial nerves}

Among the motor neurons innervating the extraocular muscles, GFP is expressed in the oculomotor (nIII) and the trochlear motor (nIV) neurons, but not in the abducens motor (nVI) neurons. GFP-positive nIII neurons are visible at $26 \mathrm{hr}$ (Fig. 5C), and subsequently, they extend axons toward the extraocular muscles (Fig. $5 D, g ; 6 B, C$ ). The projection pattern of the GFP-labeled axons to target extraocular muscles are described in Table 1. GFP-positive nIV neurons appear at 30-32 hr (data not shown). Their axons extend dorsally within the midbrain (Fig. $5 F, n$ ), cross the dorsal midline (Fig. 7A) before they exit the CNS, extend ventrally in the periphery (Fig. $6 B, C$ ), and then turn anteriorly toward their target, the superior oblique (so) muscle (Fig. 7 $A, B)$.

In the glossopharyngeal (IX) and vagus (X) cranial systems, GFP is expressed in the glossophayngeal sensory ganglion cells (Fig. 4E, gs), the vagus motor (nX) neurons, and the vagus sensory ganglion cells, which form clusters associated with each gill (vs; Figs. $4 E, 9 D$ ). No GFP expression is detected in the 
glossopharyngeal motor (nIX) neurons. By 36 hr, GFPexpressing cells in the glossopharyngeal ganglion along with their centrally projecting axons can be observed (Fig. $5 F$, $g s$ ). Later in development, peripherally extending axons into the first gills are also observed (Figs. 6B,C,E, 7C, IX ). The $\mathrm{nX}$ motor neurons form a large cluster in the posterior part of the hindbrain (Fig. $4 C, E$; Chandrasekhar et al., 1997) and extend thick axon bundles into the periphery (Fig. $5 F$ ). The pathways of the vagus sensory axons extending in both central and peripheral directions appear to coincide with the branchial (to gills) and visceral motor pathways (Fig. $7 C$; $g$ shows fibers extending into the visceral organs). Thus, the labeled tenth nerves are likely to include both motor and sensory components. Later in development, GFP is also expressed in cells other than motor neurons in the brain (Figs. $6 C$, thin arrows; $7 A, a, B, b, d ; 9 E$, single and double asterisks).

\section{DISCUSSION}

This study reports the generation of a line of transgenic zebrafish, the Isl1-GFP fish, that expresses GFP in the cranial motor neurons, some of the cranial sensory neurons, and several other groups of cells. The transparency of zebrafish embryos and larvae enabled us to obtain high-resolution images of cranial motor neurons and their axonal trajectories in intact animals throughout development. Observations of the development of the cranial motor and sensory neurons in the transgenic zebrafish were easy and reproducible. Taking advantage of these features, we first followed overall development of the $\mathrm{nV}$ neurons. Next, we have examined whether the segmental origin of the $\mathrm{nV}$ neurons correlates with target selection. Additionally, we characterized several other groups of GFP-positive neurons. The generation of this transgenic line and the description of axonal outgrowth by the GFP-positive neurons derived from analysis of this line set the stage for future experimental and genetic examination of the development of these neurons.

\section{Identification of the promoter/enhancer region of the Isl1 gene and generation of the transgenic fish}

In transient expression assays, we identified two enhancer elements of the Isll gene: one for expression in cranial motor neurons and the other for expression in Rohon-Beard cells and the trigeminal ganglion cells. Using the former element, we generated a stable transgenic line. Our strategy in dissecting the enhancer regions was as follows. First, we prepared a core plasmid that has a minimum promoter of the Isl1 gene sufficient only for expression in hatching gland cells. Next, we generated a series of DNA constructs in which different genomic fragments near the Isll gene were inserted into the core plasmid and examined enhancer activity by injecting each construct into zebrafish embryos. The ease of transient expression assays in zebrafish embryos made it feasible to test a number of DNA constructs. This approach should be generally applicable to the identification of enhancer elements of any gene that controls tissue-specific gene expression and to the subsequent generation of stable transgenic fish lines.

\section{Segmental origins within the $\mathrm{nV}$ neurons define their target fields}

Our analysis of the fifth nerve by branch-specific DiI labeling revealed that neurons supplying axons into an intermediate branch (innervating the am) are located in the anterior cluster of the $\mathrm{nV}$, and neurons supplying axons into proximal (innervating the lap and do) and distal (innervating the ima and imp) branches are located in the posterior cluster of the $\mathrm{nV}$. The am, the only muscle innervated by the anteriorly located neurons, functions as a jaw "closer" acting antagonistically with the rest of jaw "openers". Thus, the closer and opener motor neurons are located in different rhombomeres.

The organization of the trigeminal motor neurons in the CNS with respect to the peripheral nerve branches has been examined in many adult vertebrates (for references, see Song and Boord, 1993). These studies suggested that, despite the wide range of morphological differences, the organization of the trigeminal system is conserved among vertebrates. All vertebrates examined so far contain equivalent proximal, intermediate, and distal branches of $\mathrm{nV}$, with motor neurons that project into the intermediate branch located as a discrete cluster at the anterior part of the trigeminal nucleus. Our results support such previous observations and reveal that the stereotyped connections between subpopulations of the trigeminal motor neurons and the opener and closer muscles are established early in development. Because GFP-positive clusters of trigeminal neurons, from the beginning, start out as discrete clusters in $\mathrm{r} 2$ and $\mathrm{r} 3$, it is likely that the neurons of the anterior and posterior clusters are born in $\mathrm{r} 2$ and $\mathrm{r} 3$, respectively. Thus, the differences between the two clusters of trigeminal motor neurons can be attributed to the segmental origin of the motor neurons; i.e., neurons born in $\mathrm{r} 2$ and $\mathrm{r} 3$ will eventually project to distinct target muscles of different physiological functions. It is possible that the anterior cluster (r2) and the posterior cluster $(\mathrm{r} 3)$ are distinct in nature at birth, presumably under the control of the regulatory genes expressed in a rhombomere-specific manner (Lumsden and Krumlauf, 1996). Conservation of both the adult trigeminal pattern (Song and Boord, 1993) and hindbrain segmentation processes during development among vertebrates (Gilland and Baker, 1993; Lumsden and Krumlauf, 1996) suggests that this idea applies to other vertebrates, including chick and mouse.

\section{Potential use of the IsI1-GFP line for neurobiology}

The Isl1-GFP zebrafish will be useful for several types of studies. For instance, it could be used to analyze the function of genes regulating motor neuron development. By injecting DNA constructs, such as dominant negative alleles of genes normally expressed by the cranial motor neurons into Isl1-GFP embryos, one could potentially examine loss of function effects on outgrowth and pathfinding by the motor axons. Live visualization of motor neurons and their axons would allow for a dynamic analysis of the effects of such molecular manipulations on pathfinding by growth cones. The ability to assay effects dynamically would increase the detectability of phenotypes because the effect of some molecular manipulations may be most apparent and informative in a dynamic analysis. Similarly, the Isl1-GFP line would be useful for cellular manipulations such as laser ablation experiments, which could ablate specific groups of trigeminal neurons or other cell types. Furthermore, the transgenic fish might be also useful for electrophysiological studies because it would allow researchers to record the activity of a GFP-labeled neuronal type reproducibly.

Because the zebrafish is genetically manipulable, the Isl1-GFP zebrafish provides an opportunity for genetic analyses of the development of neurons such as the cranial motor neurons. Recent large-scale mutant screens have identified many mutations that cause defects in CNS or craniofacial development (Driever et al., 1996; Haffter et al., 1996). The Isl1-GFP fish will enable further examination of these mutations after introduction of the Isl1-GFP transgene into the mutant background by crossing mu- 
tant with Isl1-GFP lines. Our detailed characterization of GFPlabeled motor and sensory neurons along with their fibers provides an anatomical basis for these studies. Finally, the Isl1-GFP line can be used as a starting strain for mutational analysis of GFP-positive neurons. The line affords the possibility of screening for new mutations that affect hindbrain segmentation and determination of specific neuronal types. Furthermore, the fact that axon outgrowth can be so readily assayed in these embryos should make it possible to screen directly for mutations that affect outgrowth, pathfinding, and synapse formation by the GFPpositive neurons.

\section{REFERENCES}

Appel B, Korzh V, Glasgow E, Thor S, Edlund T, Dawid IB, Eisen JS (1995) Motoneuron fate specification revealed by patterned LIM homeobox gene expression in embryonic zebrafish. Development 121:4117-4125.

Balak KJ, Corwin JT, Jones JE (1990) Regenerated hair cells can originate from supporting cell progeny: evidence from phototoxicity and laser ablation experiments in the lateral line system. J Neurosci 10:2502-2512.

Burrill JD, Easter Jr S (1994) Development of the retinofugal projections in the embryonic and larval zebrafish (Brachydanio rerio). J Comp Neurol 346:583-600.

Chandrasekhar A, Moens CB, Warren Jr J, Kimmel CB, Kuwada JY (1997) Development of branchiomotor neurons in zebrafish. Development 124:2633-2644.

Chandrasekhar A, Warren Jr JT, Takahashi K, Schauerte HE, van Eeden FJ, Haffter P, Kuwada JY (1998) Role of sonic hedgehog in branchiomotor neuron induction in zebrafish. Mech Dev 76:101-115.

Chandrasekhar A, Schauerte HE, Haffter P, Kuwada JY (1999) The zebrafish detour gene is essential for cranial but not spinal motor neuron induction. Development 126:2727-2737.

Coombs S, Görner P, Münz H (1989) The mechanosensory lateral line: neurobiology and evolution. New York: Springer.

Driever W, Solnica-Krezel L, Schier AF, Neuhauss SC, Malicki J, Stemple DL, Stainier DY, Zwartkruis F, Abdelilah S, Rangini Z, Belak J, Boggs C (1996) A genetic screen for mutations affecting embryogenesis in zebrafish. Development 123:37-46.

Dynes JL, Ngai J (1998) Pathfinding of olfactory neuron axons to stereotyped glomerular targets revealed by dynamic imaging in living zebrafish embryos. Neuron 20:1081-1091.

Ericson J, Thor S, Edlund T, Jessell TM, Yamada T (1992) Early stages of motor neuron differentiation revealed by expression of homeobox gene Islet-1. Science 256:1555-1560.

Gilland E, Baker R (1993) Conservation of neuroepithelial and mesodermal segments in the embryonic vertebrate head. Acta Anat 148:110-123.

Guthrie S (1996) Patterning the hindbrain. Curr Opin Neurobiol 6:41-48.

Haffter P, Granato M, Brand M, Mullins MC, Hammerschmidt M, Kane DA, Odenthal J, van Eeden FJ, Jiang YJ, Heisenberg CP, Kelsh RN, Furutani-Seiki M, Vogelsang E, Beuchle D, Schach U, Fabian C, Nüsslein-Volhard C (1996) The identification of genes with unique and essential functions in the development of the zebrafish, Danio rerio. Development 123:1-36.

Hatta K, Schilling TF, BreMiller RA, Kimmel CB (1990) Specification of jaw muscle identity in zebrafish: correlation with engrailedhomeoprotein expression. Science 250:802-805.

Heaton MB, Moody SA (1980) Early development and migration of the trigeminal motor nucleus in the chick embryo. J Comp Neurol 189:61-99.

Higashijima S, Nose A, Eguchi G, Hotta Y, Okamoto H (1997a) Mindin/ F-spondin family: novel ECM proteins expressed in the zebrafish embryonic axis. Dev Biol 192:211-227.

Higashijima S, Okamoto H, Ueno N, Hotta Y, Eguchi G (1997b) Highfrequency generation of transgenic zebrafish which reliably express GFP in whole muscles or the whole body by using promoters of zebrafish origin. Dev Biol 192:289-299.
Highstein SM (1991) The central nervous system efferent control of the organs of balance and equilibrium. Neurosci Res 12:13-30.

Inoue A, Takahashi M, Hatta K, Hotta Y, Okamoto H (1994) Developmental regulation of islet-1 mRNA expression during neuronal differentiation in embryonic zebrafish. Dev Dyn 199:1-11.

Kimmel CB, Ballard WW, Kimmel SR, Ullmann B, Schilling TF (1995) Stages of embryonic development of the zebrafish. Dev Dyn 203:253-310.

Korzh V, Edlund T, Thor S (1993) Zebrafish primary neurons initiate expression of the LIM homeodomain protein Isl-1 at the end of gastrulation. Development 118:417-425.

Long Q, Meng A, Wang H, Jessen JR, Farrell MJ, Lin S (1997) GATA-1 expression pattern can be recapitulated in living transgenic zebrafish using GFP reporter gene. Development 124:4105-4111.

Lumsden A, Krumlauf R (1996) Patterning the vertebrate neuraxis. Science 274:1109-1115.

Metcalfe WK, Kimmel CB, Schabtach E (1985) Anatomy of the posterior lateral line system in young larvae of the zebrafish. J Comp Neurol 233:377-389.

Murray MJ, Merritt DJ, Brand AH, Whitington PM (1998) In vivo dynamics of axon pathfinding in the Drosophila CNS: a time- lapse study of an identified motorneuron. J Neurobiol 37:607-621.

Okada A, Lansford R, Weimann JM, Fraser SE, McConnell SK (1999) Imaging cells in the developing nervous system with retrovirus expressing modified green fluorescent protein. Exp Neurol 156:394-406.

Osumi N, Hirota A, Ohuchi H, Nakafuku M, Iimura T, Kuratani S, Fujiwara M, Noji S, Eto K (1997) Pax-6 is involved in the specification of hindbrain motor neuron subtype. Development 124:2961-2972.

Pfaff S, Kintner C (1998) Neuronal diversification: development of motor neuron subtypes. Curr Opin Neurobiol 8:27-36.

Roberts BL, Meredith GE (1989) The efferent system. In: The mechanosensory lateral line (Coombs S, Görner P, Münz H, eds), pp 445460. New York: Springer.

Roberts BL, Meredith GE (1992) The efferent innervation of the ear: variations on an enigma. In: The evolutionary biology of hearing (Webster DB, Fay RR, Popper AN, eds), pp 185-210. New York: Springer.

Schilling TF, Kimmel CB (1997) Musculoskeletal patterning in the pharyngeal segments of the zebrafish embryo. Development 124: 2945-2960.

Simon H, Guthrie S, Lumsden A (1994) Regulation of SC1/DMGRASP during the migration of motor neurons in the chick embryo brain stem. J Neurobiol 25:1129-1143.

Somiya H (1987) Dynamic mechanism of visual accommodation in teleosts: structure of the lens muscle and its nerve control. Proc R Soc Lond B Biol Sci 230:77-91.

Song J, Boord RL (1993) Motor components of the trigeminal nerve and organization of the mandibular arch muscles in vertebrates. Phylogenetically conservative patterns and their ontogenetic basis. Acta Anat 148:139-149.

Tokumoto M, Gong Z, Tsubokawa T, Hew CL, Uyemura K, Hotta Y, and Okamoto H (1995) Molecular Heterogeneity among primary motoneurons and within myotomes revealed by the differential mRNA expression of novel Islet-1 homologs in embryonic zebrafish. Dev Biol 171:578-589.

Trevarrow B, Marks DL, Kimmel CB (1990) Organization of hindbrain segments in the zebrafish embryo. Neuron 4:669-679.

Tsuchida T, Ensini M, Morton SB, Baldassare M, Edlund T, Jessell TM, Pfaff SL (1994) Topographic organization of embryonic motor neurons defined by expression of LIM homeobox genes. Cell 79:957-970.

Varela-Echavarría A, Pfaff SL, Guthrie S (1996) Differential expression of LIM homeobox genes among motor neuron subpopulations in the developing chick brain stem. Mol Cell Neurosci 8:242-257.

Westerfield M (1995) The zebrafish book. Eugene, OR: University of Oregon.

Whitfield TT, Granato M, van Eeden FJ, Schach U, Brand M, FurutaniSeiki M, Haffter P, Hammerschmidt M, Heisenberg CP, Jiang YJ, Kane DA, Kelsh RN, Mullins MC, Odenthal J, Nüsslein-Volhard C (1996) Mutations affecting development of the zebrafish inner ear and lateral line. Development 123:241-254. 